\title{
Flora of Kemaliye (Erzincan) District
}

\author{
Kemaliye İlçesinin (Erzincan) Florası
}

\author{
Research Article
}

Mehmet Ufuk Özbek ${ }^{1 *}$, Sırrı Yüzbaşıoğlu² ${ }^{2}$ Haşim Altınözlü ${ }^{3}$, Ali Kandemir $^{4}$

'Department of Biology, Faculty of Science, Gazi University, Ankara, Turkey.

Department of Pharmaceutical Botany, Faculty of Pharmacy, Istanbul University, Istanbul, Turkey.

${ }^{3}$ Department of Biology, Faculty of Science, Hacettepe University, Beytepe, Ankara, Turkey.

${ }^{4}$ Department of Biology, Faculty of Arts and Sciences, Erzincan University, Erzincan, Turkey.

\section{A B S TR AC T}

T

his research was carried out in Kemaliye district which about 158 km south-west of Erzincan province. 1800 plant specimens were collected from the area between 2005-2007. Identification of the specimens revealed the presence of 640 taxa belonging to 318 genera in 80 families. Cryptogamae includes 4, Spermatophyta includes 636 taxa (Gymnospermae 3, and Angiospermae 633 taxa). 36 taxa are new records for B7 square. A total of 118 taxa determined in the research area $(18,43 \%)$ are endemic for Turkey. The scattering ratios of the taxa for the phytogeographical regions are as follows: Irano-Turanian elements are 252 taxa (39,37\%), Mediterranean elements are 34 taxa (5,31\%), Euro.-Sib. elements are 32 taxa (5\%), widespread or other taxa which their regions unclarified are $322(50,31 \%)$.

\section{Key Words}

Flora, Kemaliye, Erzincan, Turkey.

\section{öz}

\begin{abstract}
A raştırma, Erzincan'ın 158 km güneybatısındaki Kemaliye ilçesi sınırları içerisinde gerçekleştirilmiştir A çalışma alanından 2005-2007 yılları arasında 1800 bitki örneği toplanmıştır. 80 familya ve 318 cinse ait, 640 tür ve türaltı takson tespit edilmiştir. Eğreltiler 4, açık tohumlular 3, kapalı tohumlular ise 633 takson içermektedir. 36 adet takson B7 karesi için yeni kayıttır. Toplam 118 takson Türkiye için endemiktir. Endemizm oranı \%18,43'dür. Taksonların fitocoğrafik bölgelere göre dağılımı ise şöyledir: 252'si İran-Turan elementi (\%39,37), 34' ü Akdeniz elementi (\%5,31); 32'si Avr.-Sib. elementi (\%5), 322' si çok bölgeli veya bölgesi belirlenemeyen $(\% 50,31)$ taksonlardır.
\end{abstract}

\section{Anahtar Kelimeler}

Flora, Kemaliye, Erzincan, Türkiye.

Article History: Received: Apr 27, 2018; Revised: Jul 7, 2018; Accepted: Oct 8, 2018; Available Online: Nov 13, 2018. DOI: $10.15671 /$ HJBC.2018.261

Correspondence to: M. U. Özbek, Department of Biology, Faculty of Science, Gazi University, Ankara, Turkey. 


\section{INTRODUCTION}

The he district Kemaliye in Erzincan is located on the northwest border of Eastern Anatolia Region. The study area is situated between $39^{\circ} 15$ $00^{\prime \prime} \mathrm{N}$ latitude and 38 $30^{\prime} 00^{\prime \prime} \mathrm{E}$ longitude. The altitude of the district is $950 \mathrm{~m}$ above sea level [1] The surface area is $1007 \mathrm{~km}^{2}$ [2]. Eastern Anatolia Region is divided into four regions. These are Erzurum-Kars, Yukarı Fırat, Yukarı Murat-Van and Hakkari divisions. Kemaliye is located in Yukarı Firat division. Kemaliye is surrounded in the east by Çemişgezek and Ovacık district (Tunceli province), in the south by Arapgir district (Malatya province) and Ağın district (Elazığ province), in the west by Divriği district (Sivas province) and in the north by iliç district (Erzincan province). The district center on the southwest of Erzincan province is located in Karasu Valley in the west of the Munzur Mountains. Karasu, the largest brach of the Firat, passes through the east of the district center. The settlement texture of the town shows a linear development in the steep and rocky slope of Karasu (Figure 1).

The Kemaliye district is located in the southern part of our country, in the northeast part of the Taurus Orogenic Valley (east of the Taurus) and in the area called Munzur Mountains. The Munzur Mountains are a mountain range, consisting of a large number of mountains and hills extending in the northeast-southwest direction and whose heights are mostly over 2000 m. Geological units in and around Kemaliye are slope rubble, limestone ophiolite and schist. [3].

In the Kemaliye district are generally found which is alluvial, colluvial, brown, brown forest soils and bare rocks and also rubble [4].

According to Kemaliye meteorological station, Kemaliye is meditarrenean climate less rainfall and take part in bioclimatic zone where winter is cold, while Erzincan is semi dry and very cold [5]. In the Kemaliye annual rainfall is about $808,6 \mathrm{~mm}$ and the seasonal precipitation regime is autumn, winter, spring and summer, while Erzincan annual rainfall is about $379.4 \mathrm{~mm}$ and the seasonal precipitation regime is spring, autumn, winter and summer [6]. The ombrothermic diagrams show dry and rainy months (Figure 2).

The aim of this research is to determine the flora of the Kemaliye and also to contribute to the Flora of Turkey.

\section{MATERIALS and METHODS}

The materials of the study consisting of 1800 vascular plant specimens were collected between

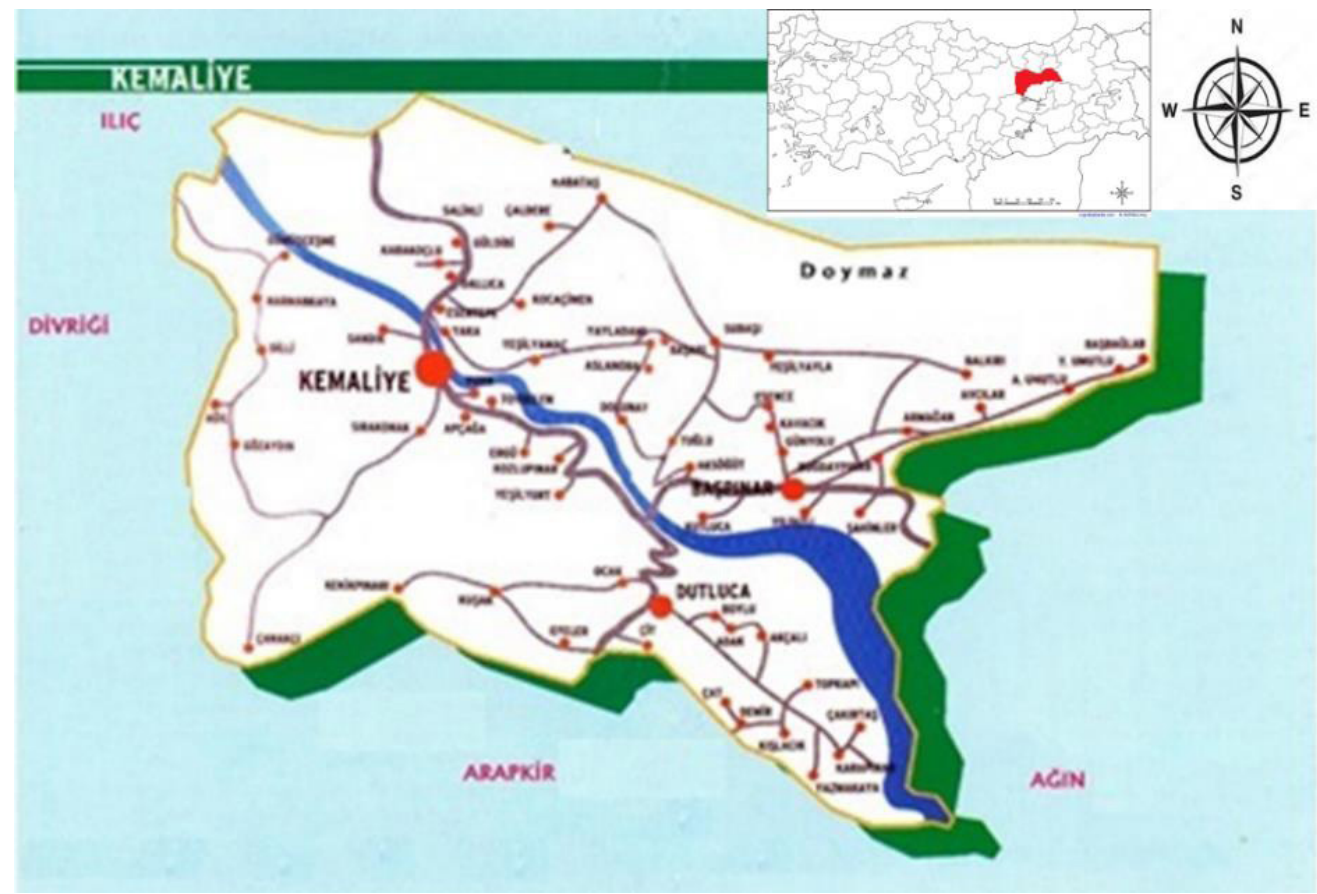

Figure 1. Map of the Kemaliye. 
a $\quad$ b $\quad$ c $\quad$ d $\quad$ e

Erzincan $850 \mathrm{~m}(33-33) 10,8^{\circ} \mathrm{C} 379,4 \mathrm{~mm}$

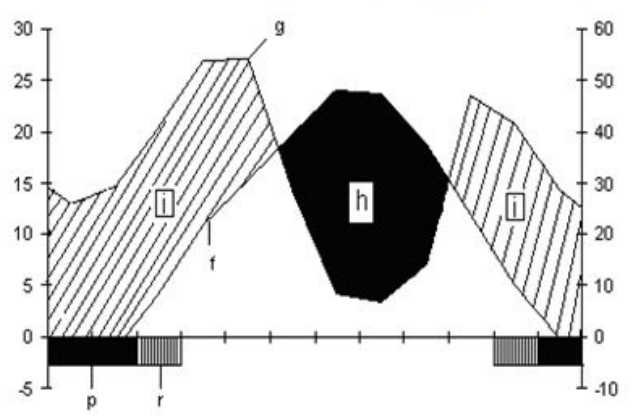

$\begin{array}{llllll}\text { a } & b & c & d & \text { e }\end{array}$

Kemaliye $950 \mathrm{~m}(6-5) \quad 13,3^{\circ} \mathrm{C} 808,6^{\circ} \mathrm{C}$

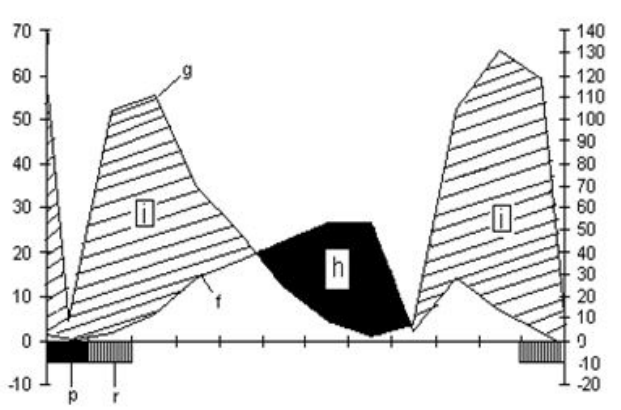

Figure 2. Ombrothermic diagrams of Erzincan and Kemaliye (a- station; b-altitude; c-observation period; $\mathbf{d}$ - mean temperature; e- annual rainfall).

2005 and 2007. During identification of the plant specimens, specimens were compared with specimens in ANK, GAZI, HUB and ISTF herbaria. The specimens are deposited in herbarium HUB and The Kemaliye Nature Museum. The most of the specimens were identified using Flora of Turkey and The East Aegean Islands [7-9] and other Floras [10-14]. All taxa in the floristic list were given according to the order in the A Checklist of the Flora of Turkey (Vascular Plants) [15]. At results, every species was represented with the following details: family and taxon name, authors of the species, Turkish name, geographical locations, habitats, altitudes, collection dates, collector numbers and endemism, IUCN threat categories $[16,17]$ and phytogeographical regions were given. Grid square and city name are not mentioned for each location because the study area is completely within the B7 Erzincan (Kemaliye). Author abbreviations of plant names were given according to Brummitt and Powell [18]. The floristic list were compared with floristic investigations of neighbouring areas [19-22].

A new record for the B7 square were determined through distribution maps in "Flora of Turkey 1-10" and other literatures [23-44]. The symbol $\left(^{*}\right)$ in front of a plant name indicates a new record for the B7 square.

For the map of the study area, the map of the painter Sıtkı Fırat was used. Relevant literatures were used for information on the geology and soil groups of the study area $[3,4]$.
Meteorological data belonging to Erzincan and Kemaliye province was obtained from Turkish State Meteorological Service [1,6]. Climate diagrams belonging to the research field were drawn according to Gaussen method. For climatic evaluation of research area the formulation of Emberger that developed for Mediterranean climate layers classification, and drought degrees was used. For the interpretation of the data about climate the book "iklim ve Biyoiklim" was used [5].

The abbreviations used in the text and the floristic list are as follows (in alphabetic order): ADK: Aslı Doğru Koca, ANK: Herbarium of Ankara University, cm: Centimeter, CR: Critically Endangered, DD: Data Deficient, EN: Endangered, End.: Endemic, E. Medit.: East Mediterranean, EuroSib.: Euro-Siberian, EX: Extinct, GAZI: Herbarium of Gazi University, HA: Haşim Altınözü, HUB: Herbarium of Hacettepe University, ISTF: Herbarium of Istanbul University, Faculty of Science, Ir.-Tur.: Irano-Turanian, IUCN: The International Union for Conservation of Nature, LC: Least Concern, Medit.: Mediterranean, m: Meter, mm: Millimeter, MK: Makbule Karahan, MUÖ: Mehmet Ufuk Özbek, MV: Mecit Vural, NT: Near Threatened, subsp.: Subspecies, SYO: Sırrı Yüzbaşıoğlu, Kandemir: Ali Kandemir, var: Variete, VU: Vulnerable.

Collecting localities in the study area as follows: 1- Kemaliye, Yeşilyamaç-Yayladamı village; 2- Kemaliye, Yeşilyamaç village; 3- Kemaliye, Yeşilyamaç-Kemaliye; 4- Kemaliye, between YakaYeşilyamaç village; 5- Kemaliye, between Yeşilyamaç- 
Yeşilyamaç stream; 6- Kemaliye, between GeşobeliYeşilyamaç; 7- Kemaliye, Yeşilyamaç-Başpınar, ahead Dolunay village; 8- Kemaliye, Dutluca, around Dutbeli; 9- Kemaliye, Yeşilyayla village, Mezra stream; 10- Kemaliye, above Yeşilyayla village; 11Kemaliye, Tozlupınar; 12- Kemaliye, between Yuva village-around Kabanbaşl; 13-Kemaliye, Yuva village; 14- Kemaliye, between Toybelen-Yuva; 15- Kemaliye, Apçaağa village, Apça vineyards; 16- Kemaliye, between Kemaliye-Apçaağa; 17- Kemaliye, between Venk bridges-Salihli village; 18 - Kemaliye, side of Venk stream; 19- Kemaliye, Bağıştaş-Kemaliye 4 km; 20- Kemaliye, between Salihli village-Bağıştaş 13. km; 21- Kemaliye, between Salihli village-Bağıştaş 17. km; 22- Kemaliye, Salihli village-Bağıştaş, 1,5 km from Ortatepe village; $23-$ Kemaliye, Salihli, çimento geçidi; 24- Kemaliye, Salihli village; 25 - Kemaliye, above Salihli village; 26- Kemaliye, above Salihli village, around stony pit; $27-$ Kemaliye, Sandık village; 28- Kemaliye, around Sandık village; 29- Kemaliye, Sandık village, around Erçivit; 30Kemaliye, Çit village; 31- Kemaliye, Sırakonaklar village; 32- Kemaliye, between SirakonaklarSubatan; 33- Kemaliye, Subatan; 34- Kemaliye, Mazbanbaşl; 35- Kemaliye, Sarıçiçek upland; 36Kemaliye, Sarıçiçek upland, around maden; 37Kemaliye, road of Sarıçiçek upland, around Mustafa Akyüz fountain; 38- Kemaliye, between SarıçiçekMazbanbaşı; 39- Kemaliye, Başpınar bridge; 40Kemaliye, Başpınar village; 41- Kemaliye, around Başpınar, above Harmankaya stream; 42- Kemaliye, Başpınar, above Konsar village; 43- Kemaliye, Keklikpınarı village; 44- Kemaliye, above Keklikpınar village; 45- Kemaliye, Sıraca village; 46- Kemaliye, above Ergü village; 47- Kemaliye, Ergü vineyards, throughout Karasu river basin; 48- Kemaliye, $5 \mathrm{~km}$ from Kuşak village; 49- Kemaliye, Kuşak village; 50Kemaliye, Taş road, Karanlık Canyon, entrance of Recep Yazıcıoğlu Tunnel; 51- Kemaliye, Taş road; 52Kemaliye, Dilli stream; 53- Kemaliye, around Avas; 54- Kemaliye, Kayıkbaşı, around fisherman hut; 55- Kemaliye, Karakoçlu; 56- Kemaliye, between Karakoçlu- Meşebaşı; 57- Kemaliye, between İkisuDavulköprü; 58- Kemaliye, above Ulaş village; 59- Kemaliye, Subaşı village; $60-$ Kemaliye, Subaşı village, around Adnan Öztürk bridge; 61- Kemaliye, Kırkgöz; 62- Kemaliye, between Kabanbaşı-Kırgöz; 63- Kemaliye, Kocaçimen village, Davulköprü; 64- Kemaliye, above Kabataş village, Karadağ; 65- Kemaliye, Munzur Mountain, Doymuş hill; 66-
Kemaliye, around Zincirkaya; 67- Kemaliye, around Alamağarası; 68- Kemaliye, Ağıllar; 69- Kemaliye, Rabat stream, throughout Karasu river basin; 70Kemaliye, Balkır village, Doymuş hill; 71- Kemaliye, Çanakçı village; 72- Kemaliye, i̇shakpaşa mahallesi; 73- Kemaliye, Kemaliye-Arapgir, $1 \mathrm{~km}$ from Yeşilyurt village signboard; 74- Kemaliye, above Yeşilyurt village; 75- Kemaliye, Arnavut inn; 76- Kemaliye, Kocahaydar, Sülük lake; 77- Kemaliye, side of governorship residence; 78- Kemaliye, Kırkgöz, radio link station; 79-Kemaliye, Armağan village; 80 Kemaliye, Binkoç village; 81- Kemaliye, $1 \mathrm{~km}$ from Sırakonaklar village

\section{RESULTS and DISCUSSION}

As a result of the examination of 1800 plants specimens which were collected from the research area between 2005-2007 years, 640 taxa that belong to 318 genera and 80 families were determined from the area. Four taxa of them belong to Pteridophyta and 636 taxa belong to Spermatophyta. The number of endemic taxa in the study area is 118 and the endemism ratio is $18,43 \%$.

The phytogeographical regions of the determined species in the study area, are listed as follows: Irano-Turanian elements 252 (39.37\%), Mediterranean elements 34 (5.31\%), Euro-Siberian elements $32(5 \%)$, and either multi-regional or unknown 322 (50.31\%) taxa. The comparison of the species's phytogeographical distributions among our and adjacent areas are presented Table 1. IranoTuranian elements seem to be dominantly in all area studied.

1. This research

2. Yıldırımlı - Flora of Munzur Mountains (ErzincanTunceli) [19]

4. Akpulat - Flora of Between Sivas-Sıcak Çermik (Sivas) [20]

5. Türkoğlu - Flora of Karga Mountain (Elazığ) Florası [21]

6. Yıldız- Floristical Characteristics of Berit Mountain (Kahramanmaraş) [22]

The threat categories of Psephellus psephelloides (Freyn. \& Sint.) Wagenitz (Syn: Centaurea psephelloides), Physocardamum 
Table 1. A comparison of the phytogeographical elements.

\begin{tabular}{llllll}
\hline \multirow{2}{*}{ Phytogeographical regions } & \multicolumn{5}{c}{ Research areas } \\
\cline { 2 - 6 } & $1(\%)$ & $2(\%)$ & $3(\%)$ & $4(\%)$ & $5(\%)$ \\
\hline Irano-Turanian & 39.37 & 45.7 & 31.3 & 32.5 & 28.2 \\
\hline Mediterranean & 5.31 & 4.4 & 5.2 & 5.1 & 15.0 \\
\hline Euro-Siberian & 5 & 8 & 5.6 & 3.7 & 5.6 \\
\hline Multi-regional or unknown & 50.31 & 41.9 & 57.9 & 58.7 & 51.2
\end{tabular}

angustifolium (Hausskn. ex Bornm.) Kandemir (Syn: Bornmuellera angustifolia) and Teucrium leucophyllum Montbret \& Aucher ex Benth. are $\mathrm{DD}$, Verbascum calycosum Hausskn ex Murb. Barbarea auriculata Hausskn. ex Bornm var. auriculata, Onosma discedens Hausskn. ex Bornm. and Onosma affinis Hausskn. ex Riedl are EX in Red Data Book of Turkish Plants [16]. Previously, these taxa were collected from the study area and threat categories of the species were proposed [45-48]. Recently, Aethionema ozbekii Yıld. had been published [49], but threat category of this species have not been determined until this study. We determined that the area of occupancy estimated to be less than $500 \mathrm{~km}^{2}$, severely fragmented or known to exist at no more than five locations and decline of number of mature individuals. Therefore, according to IUCN Red List criteria [49], category of the threatened Aethionema ozbekii is proposed as EN B2ab(v). The results are summarized in Table 2.
The total number of endemic taxa is 118 (18.43\%). The endemism rate is $275(18.11 \%)$ for Flora of Munzur Mountains (Yıldırımlı, 1995), 61 (17.8\%) for Flora of Between Sivas-Sıcak Çermik (Akpulat \& Çelik, 2002), 200 (17.16\%) for Floristical Characteristics of Berit Mountain (Yıldız, 2001) and 46 (9\%) Flora of Karga Mountain (Türkoğlu \& Civelek, 2005) (Table 3).

The families including the highest numbers of the taxa in research field are as follows: Asteraceae 86 (\%13.43), Brassicaceae 62 (\%9.68), Fabaceae 60 (\%9.37), Lamiaceae 55 (\%8.59), Caryophyllaceae 33 (\%5.15), Apiaceae 30 (\%4.68), Boraginaceae 29 (\%4.53), Rosaceae 24 (\%3.75), Poaceae 16 (\%2.5) and Caprifoliaceae 15 (\%2.34) (Table 4). Other 230 taxa left belong to other families.

The genus Astragalus includes the highest number of species in research area with 13 taxa. Alyssum and Silene (11), Centaurea (10), Papaver

Table 2. The numbers and rates of the threatened species and IUCN categories.

\begin{tabular}{ccc}
\hline Category & Species number & Ratio to total endemic number (\%) \\
\hline CR & 9 & 7.82 \\
\hline EN & 11 & 9.56 \\
\hline VU & 14 & 11.17 \\
\hline NT & 15 & 13.04 \\
\hline LC & 66 & 57.39 \\
\hline
\end{tabular}

Table 3. A comparison of the number of endemic taxa and endemism rate among our and adjacent studies.

\begin{tabular}{lccccc}
\hline \multirow{2}{*}{ Endemism } & \multicolumn{5}{c}{ Research areas } \\
\cline { 2 - 6 } & 1 & 2 & 3 & 4 & 5 \\
\hline The number of endemic taxa & 118 & 275 & 61 & 46 & 200 \\
\hline Endemism rate (\%) & 18.43 & 18.11 & 17.8 & 9 & 17.16 \\
\hline Total number of taxa & 640 & 1518 & 341 & 510 & 1165 \\
\hline
\end{tabular}


Table 4. Families and their rates (\%) including the highest number of the taxa.

\begin{tabular}{lcclcc}
\hline \multicolumn{1}{c}{ Family } & Number of the taxa & Rate (\%) & Family & Number of the taxa & Rate (\%) \\
\hline Asteraceae & 86 & 13.43 & Apiaceae & 30 & 4.68 \\
\hline Brassicaceae & 62 & 9.68 & Boraginaceae & 29 & 4.53 \\
\hline Fabaceae & 60 & 9.37 & Rosaceae & 24 & 3.75 \\
\hline Lamiaceae & 55 & 8.59 & Poaceae & 16 & 2.5 \\
\hline Caryophyllaceae & 33 & 5.15 & Caprifoliaceae & 15 & 2.34 \\
\hline
\end{tabular}

and Onosma (9), Aethionema, Scorzonera and Medicago (8), Salvia and Thymus (7), Anthemis, Taraxacum and Allium (6) taxa follow (Table 5).

According to the Flora of Turkey, the richest families in terms of taxa numbers are Asteraceae, Fabaceae, Lamiaceae, Brassicaceae, Caryophyllaceae, Scrophulariaceae, Apiaceae, Liliaceae and Boraginaceae. In our study, Asteraceae, Brassicaceae and Fabaceae families are ordered as the richest families in terms of taxa number. The comparison of the richest families at taxa numbers level among this research and adjacent studies is given in Table 6 .

Floristic list is given below;
1. PTERIDOPHYTA / EĞRELTI BÖLÜMÜ

ASPLENIACEAE / SAÇAKOTUGILLER

Asplenium ceterach L. "dalakotu", 4, rocky places, 1040 m., 16.05.2006, HA 5303.

CYSTOPTERIDACEAE

\section{GEVREKEĞRELTIGILLER}

Cystopteris fragilis (L.) Bernh. "gevrek eğrelti", 33, calcareous rocky places, 1850 m., 17.05.2006, HA 5334.

\section{EQUISETACEAE / ATKUYRUĞUGILLER}

Equisetum giganteum L. "kırkkilitotu”, 43, damp area, 1400 m., 15.05.2006, HA 5233.

PTERIDACEAE / BALDIRIKARAGILLER

Table 5. Genera including the highest number of taxa and their rates.

\begin{tabular}{lcclcc}
\hline Genus & $\begin{array}{c}\text { Number } \\
\text { of the } \\
\text { taxa }\end{array}$ & Rate (\%) & Genus & $\begin{array}{c}\text { Number of } \\
\text { the taxa }\end{array}$ & Rate (\%) \\
\hline Astragalus & 13 & 2.03 & Scorzonera & 8 & 1.25 \\
\hline Alyssum & 11 & 1.71 & Medicago & 8 & 1.25 \\
\hline Silene & 11 & 1.71 & Salvia & 7 & 1.09 \\
\hline Centaurea & 10 & 1.56 & Thymus & 7 & 1.09 \\
\hline Papaver & 9 & 1.4 & Anthemis & 6 & 0.93 \\
\hline Onosma & 9 & 1.4 & Taraxacum & 6 & 0.93 \\
\hline Aethionema & 8 & 1.25 & Allium & 6 & 0.93 \\
\hline
\end{tabular}

Table 6. Comparison of the richest families in terms of taxa numbers in research area other studies in neighbourhood.

\begin{tabular}{lccccc}
\hline Research area & 1 & 2 & 3 & 4 & 5 \\
\hline Family & $\begin{array}{c}\text { Number } \\
\text { of the } \\
\text { taxa }\end{array}$ & $\begin{array}{c}\text { Number } \\
\text { of the } \\
\text { taxa }\end{array}$ & $\begin{array}{c}\text { Number } \\
\text { of the } \\
\text { taxa }\end{array}$ & $\begin{array}{c}\text { Number } \\
\text { of the } \\
\text { taxa }\end{array}$ & $\begin{array}{c}\text { Number } \\
\text { of the } \\
\text { taxa }\end{array}$ \\
\hline Asteraceae & 86 & 171 & 53 & 59 & 144 \\
\hline Brassicaceae & 62 & 121 & 40 & 45 & 77 \\
\hline Fabaceae & 60 & 70 & 41 & 65 & 130 \\
\hline Lamiaceae & 55 & 101 & 30 & 28 & 73 \\
\hline Caryophyllaceae & 33 & 129 & 20 & 25 & 75 \\
\hline
\end{tabular}


Cheilanthes persica (Bory) Mett. ex Kuhn, "paslı eğrelti", 77, rocky places, 870 m., 14.05.2006, MUÖ 1970.

\section{PINOPHYTINA / AÇIK TOHUMLULAR}

\section{EPHEDRACEAE / DENIZÜZÜMÜGILLER}

Ephedra major Host, "hum", 61, steppe, 1260 m., 12.07.2006, MV 9176, E. Medit.

CUPRESSACEAE / SERVIGILLER

Juniperus oxycedrus $L$. subsp. oxycedrus "katran ardıcı", 40, Juniperus and Quercus mixed area, 875 m., 16.05.2006, HA 5258.

J. excelsa M. Bieb. subsp. excelsa, "boz ardıç", 7 , rocky places, 1485 m., 24.09.06, SYO 2837.

\section{MAGNOLIOPHYTINA / KAPALI TOHUMLULAR}

\section{ACANTHACEAE / AYIPENÇESIGILLER}

Acanthus dioscoridis $\mathrm{L}$. var. dioscoridis, "lokman ayıpençesi", 1, stony slopes, 1600 m., 13.07.2006, MV 9246.

\section{AMARANTHACEAE / HOROZIBiĞiGILLER} Chenopodium botrys L. "kızllbacak", 34, roadsides, 1830 m., 23.09.06, SYO 2825.

C. foliosum Asch. "cülek", 6, rocky slopes, 1200 m., 20.10.2006, HA 5321.

Noaea mucronata (Forssk.) Asch. \& Schweinf. subsp. mucronata, "hölmezotu", 63, rocky places, 1360 m., 14.09.2007, SYO 2979.

\section{AMARYLLIDACEAE / NERGISGILLER}

Allium armenum Boiss. \& Kotschy, "pempe sırım", 33, rocky places, 1870 m., 07.07.2006, SYO 2734, End., LC, Ir.-Tur.

A. asperiflorum Miscz. "benekli soğan", 33, high mountain steppe, 1885 m., 11.06.2006, MUÖ 2060, End., LC, Ir.-Tur.

A. chrysantherum Boiss. \& Reut. "sarıkafa", 17, Quercus fields, 912 m., 14.05.2006, HA 5028, Ir.Tur.

A. kharputense Freyn \& Sint. "harput soğanı", 13 meadows, 900 m., 15.04.2006, MV 9573, Ir.-Tur.

A. scorodoprasum L. subsp. rotundum (L.) Stearn, "deli pırasa", 43, cultivated area, 1277 m. 10.06.2006, MUÖ 2046.

A. sivasicum Özhatay \& Kollman, "sivas soğanı", 23, serpentine, 1467 m., 05.07.2005, Kandemir 7013, End., LC, Ir.-Tur.

Sternbergia clusiana (Ker-Gawl.) Ker-Gawl. ex
Spreng. "vargetgülü", 37, rocky places, 1770 m., 23.09.2006, SYO 2823, Ir.-Tur.

ANACARDIACEAE / MENENGIÇGILLER

Pistacia terebinthus L. subsp. terebinthus, "menengiç", 3, roadsides, rocky slopes, 1090 m., 12.06.2006, MUÖ 2103, E. Medit.

Rhus coriaria L. "sumak", 15, Quercus fields, 23.09.2006, SYO 2820.

\section{APIACEAE / MAYDANOZGILLER}

Antriscus nemorosa (M. Bieb.) Spreng. "peçek", 31, meadows, 1200 m, 14.04.2006, MV 9384.

Artedia squamata L. "karabenek", 4, serpentine stony slopes, 915 m, 13.07.2006, MV 9192.

Astrodaucus orientalis (L.) Drude, "havyıldız", 21, 1130 m., 04.07.2007, MUÖ 2544, Ir.-Tur.

Bunium elegans (Fenzl) Freyn var. elegans, "hoş aksar", 78, stony slopes, 1800 m, 23.05.2014, Kandemir 10499.

Bunium elegans (Fenzl) Freyn var. involucratum Ö.Saya, "hoş aksar", 78, stony slopes, 1800 m, 23.05.2014, Kandemir 10500, End., CR, Ir.-Tur.

Bupleurum eginense (Wollf) Snogerup, "eğin şeytanayağı", 20, rocky slopes, 1270 m., 04.07.2007, MUÖ 2526, End., NT.

Cnidium silaifolium (Jacq.) Simonk. subsp. orientale (Boiss.) Tutin, "galyobişotu", 46, rocky places, 1230 m., 05.07.2007, MUÖ 2577.

Eryngium billardieri F. Delaroche, "hıyarok", 30, rocky places, 940 m., 13.09.2007, SYO 2968, Ir.Tur.

Falcaria vulgaris Bernh. "orakotu", 77, open stony areas, 1724 m., 15.09.2007, SYO 2993.

Ferula brevipedicellata Peşmen ex Sağıroğlu \& H. Duman, "asaotu", 25, serpentine stony slopes, 1460 m, 13.07.2005, MV 9245, End., VU.

Grammosciadium daucoides DC. "kami", 39, rocky places, 870 m, 15.05.2006, HA 5143, Ir.-Tur. Heptatera anisoptera (DC.) Tutin, "kanatlı çakşır", 43, cultivated area, 1277 m, 10.06.2006, MUÖ 2043.

Johrenia dichotoma DC. "Irazdene", 45, stony slopes, 1150 m, 12.07.2005, MV 9152, Ir.-Tur.

Malabaila secacul (Mill.) Boiss. "davarotu", 48, edge of stream, rocky slopes, $1025 \mathrm{~m}, 10.06 .2006$, MUÖ 2028.

Opopanax hispidus (Friv.) Griseb, "kaymacık", 46, rocky places, 1230 m., 05.07.2007, MUÖ 2573.

Physocaulis nodosus (L.) Tausch, "hacıkuş", 50, rocky places, $1200 \mathrm{~m}, 16.05 .2006$, HA 5310.

*Pimpinella cappadocica Boiss. \& Balansa var. 
cappadocica, "peri anasonu", 25, rocky slopes 1170 m., 02.07.2007, MUÖ 2498, End., LC, Ir.-Tur. P. kotschyana Boiss. "peri anasonu", 2, rocky slopes, 1686 m, 08.07.2006, SYO 2781, Ir.-Tur.

$\boldsymbol{P}$. peucedanifolia Fisch. "elmas süpürgesi", 23, serpentine, 1467 m, 05.07.2005, Kandemir 7006, Ir.-Tur.

P. tragium Vill. subsp. lithophila (Schischk.) Tutin, "teke anasonu", 52, rocky places, $890 \mathrm{~m}$. 04.07.2007, MUÖ 2564.

P. tragium Vill. subsp. pseudotragium (DC.) V.A Matthews, "yoz anason", 32, stony slopes, $1450 \mathrm{~m}$ 12.07.2005, MV 9132, Ir.-Tur.

Prangos meliocarpoides Boiss. var. meliocarpoides, "sultan teresi", 20, rocky slopes, 1270 m., 04.07.2007, MUÖ 2536, End., LC, Ir.-Tur. P. pabularia Lindl. "beyik", 48, edge of stream, rocky slopes, 1025 m, 10.06.2006, MUÖ 2040, Ir.Tur.

Rhabdosciadium urusakii Akalın, "iliç handoğu", 23, serpentine, roadsides, 950 m, 23.07.2015, Kandemir 10844, End., CR, Ir.-Tur.

Scandix stellata Banks \& Sol. "dağ kişnişi", 13, cultivated area, 900 m, 15.04.2006, MV 9561.

*Smyrnium connatum Boiss. \& Kotschy, "yabani kereviz", 4, rocky places, 1040 m, 16.05.2006, HA 5293, E. Medit.

Torilis leptocarpa (Hochst.) C.C. Towns. "narin dercikotu", 32, stony slopes, 1190 m, 12.07.2005, MV 9119, Ir.-Tur.

Trigonosciadium intermedium Freyn \& Sint. ex Sint., "öz şemsiye otu", 81, edge of fields, 13.06.2013, 970 m, Kandemir 10393, End., CR, Ir.Tur.

Turgenia latifolia (L.) Hoffm. "karaheci", 43, cultivated area, 1277 m, 10.06.2006, MUÖ 2044.

*Turgeniopsis foeniculacea (Fenzl) Boiss. "demirpitrak", 41, Juniperus and Quercus mixed area, 1030 m, 16.05.2006, HA 5254.

\section{APOCYNACEAE / ZAKKUMGILLER}

Cionura erecta (L.) Griseb. "babrik", 54, roadsides, 861 m, 04.06.2007, HA 5625, E. Medit.

Periploca graeca L. var. graeca, "garipler urganı", 15, Quercus fields, 23.09.2006, SYO 2819, HA 5624, E. Medit.

Vinca herbacea Waldst. \& Kit. "bikir çiçeği", 53, serpentine slopes, 1500 m., 14.05.2006, HA 5069. V. sonerii Koyuncu, "osman çiçeği", 4, serpentine stony slopes, 915 m, 13.07.2005, MV 9186, End., LC, Ir.-Tur.

Vincetoxicum canescens (Willd.) Decne. subsp. canescens, "zilasur", 32, stony slopes, 1490 m., 12.07.2006, MV 9138, Ir.-Tur.

V. fuscatum (Hornem.) Rchb. f. subsp. fuscatum, "gavur biberi", 55, Quercus fields, 1287 m., 14.05.2006, HA 5089.

\section{ARACEAE / YILANYASTIĞIGILLER}

Arum elongatum Steven, "yılancücüğü", 34, rocky places, 1670 m., 17.05.2005, HA 5321, Ir.-Tur. *Spirodela polyrhiza (L.) Schleid. "telli sumercimeği", 76, slough, 1724 m., 15.09.2007, SYO 2990.

ARISTOLOCHIACEAE / LOHUSAOTUGILLER

Aristolochia bottae Jaub. \& Spach, "köpektaşağı", 11, rocky slopes, 1150 m, 15.05.2006, HA 5217, Ir.Tur.

\section{ASPARAGACEAE / KUSKONMAZGILLER}

Bellevalia gracilis Feinburn, "aktepeli", 17, Quercus fields, 912 m., 14.05.2006, HA 5041, End., LC, Ir.-Tur.

Hyacinthella acutiloba K. Perss. \& Wendelbo, "sivrisümbül" 6, rocky places, 1680 m., 13.04.2006, MV 9345, End., LC, Ir.-Tur.

*Hyacinthus orientalis L. subsp. chionophilus Wendelbo, "kopça", 6, roadsides, 1400 m., 13.04.2006, MV 9367, End., NT, Ir.-Tur.

Muscari azureum Fenzl, "keşişbaşı", 34, rocky slopes, 1650 m., 14.04.2006, MV 9370, End., LC. M. armeniacum Leichtlin ex Baker, "gavurbaşı", 31, meadows, 1200 m., 14.04.2006, MV 9382.

M. coeleste Fomin, "kediboncuğu", 34, rocky slopes, 1650 m, 14.04.2006, MV 9371, End., LC, Ir.-Tur.

Ornithogalum arcuatum Steven, "kurtkirişi", 11, rocky places, 1150 m., 15.05.2006, HA 5215, Ir.-Tur. O. oligophyllum E.D. Clarke, "kurtsoğanı", 34, rocky slopes, 1650 m., 14.04.2006, MV 9379.

* O. orthophyllum Ten. subsp. kochii (Parl.) Maire \& Weiller, "bayır yıldızı", 34, rocky places, 1670 m., 17.05.2006, HA 5313.

0. sphaerocarpum A. Kern. "salkım sakarca", 17, Quercus fields, 912 m., 14.05.2006, HA 5044.

Scilla sibirica Haw. subsp. armena (Grossh.) Mordak, "camışkıran", 34, rocky slopes, 1650 m., 14.04.2006, MV 9369, Ir.-Tur.

\section{ASTERACEAE / PAPATYAGILLER}

Achillea aleppica DC. subsp. aleppica, "tatarcI otu", 39, rocky places, 870 m., 15.05.2006, HA 5160, Ir.-Tur.

A. magnifica Heimerl ex Hub. -Mor. "karcivanı", 56, rocky places, 1715 m., 09.07.2006, SYO 2793, End., NT, Ir-Tur. 
A. schischkinii Sosn. "deli civanperçimi", 34, rocky places, 1630 m, 02.06.2007, HA 5453, End., LC, Ir-Tur.

A. teretifolia Willd. "beyaz civanperçimi", 36, 1620 m., 05.07.2005, Kandemir 7033, End., LC, Ir.-Tur.

Anthemis cretica L. subsp. anatolica (Boiss.) Grierson, "horoz papatyası", 26, rocky places, 1380 m, 04.07.2007, MUÖ 2557.

A. cretica L. subsp. pontica (Willd.) Grierson, "laz papatyası", 25, serpentine slopes, 1400 m, 13.07.2005, MV 9205.

A. kotschyana Boiss. var. discoidea (Bornm.) Grierson, "koç papatyası", 25, rocky slopes, 1460 m., 13.07.2005, MV 9227.

A. kotschyana Boiss. var. kotschyana, "koç papatyası", 20, rocky slopes, $1270 \mathrm{~m} ., 04.07 .2007$, MUÖ 2532.

A. kotschyana Boiss. var. radians Bornm. "koç papatyası", 38, roadsides, rocky slopes, $1650 \mathrm{~m}$. 11.06.2006, MUÖ 2076.

*A. pungens Yavin, "geyik papatyası", 13, cultivated area, 900 m., 15.04.2006, MV 9507, End., NT.

Arctium minus (Hill) Bernh. "löşlek", 30, roadsides, forest clearings, $1030 \mathrm{~m}$., 06.07.2006, SYO 2686, Euro-Sib.

Carduus nutans L. subsp. nutans, "eşekdikeni", 36, rocky places, 1875 m, 07.07.2006, SYO 2739.

Carlina oligocephala Boiss. \& Kotschy subsp. oligocephala, "domuz dikeni", 30, rocky slopes, 940 m., 13.09.2007, SYO 2970.

Centaurea aggregata Fisch. \& C.A. Mey. ex DC. subsp. aggregata, "kümedüğme", 33, stony slopes, 1190 m., 12.07.2005, MV 9120.

C. aucheri (DC.) Wagenitz, "güdük sarıbaş", 8, Quercus fields, 1225 m., 05.07.2007, MUÖ 2583, End., EN, Ir.-Tur.

C. carduiformis DC. subsp. carduiformis, "kavgalaz", 28, rocky slopes, 1170 m., 02.07.2007, MUÖ 2476.

C. consanguinea DC. "tezdüğme", 4, stony slopes, 915 m., 13.07.2005, MV 9190, End., EN, Ir.-Tur.

C. derderiifolia Wagenitz, "bağ sarıbaşı", 59, rocky places, 1538 m, 25.09.2006, SYO 2850, End., NT Ir.-Tur.

C. drabifolia Sibth. \& Sm. subsp. drabifolia, "öbek sarıbaş", 1, stony slopes, 1600 m., 13.07.2005, MV 9260.

C. polypodifolia Boiss. var. pseudobehen (Boiss.) Wagenitz, "akbehmen", 28, rocky slopes, 1170 m. 02.07.2007, MUÖ 2481, Ir.-Tur.
C. solstitialis L. subsp. solstitialis, "çakırdikeni", 32, stony slopes, 1190 m., 12.07.2005, MV 9124.

C. urvillei DC. subsp. urvillei, "alakötürüm", 42, steppe, 1468 m, 04.06.2007, HA 5640, E. Medit. C. virgata Lam. "acı süpürge", 61 , stony slopes, 1280 m., 12.07.2005, MV 9166, Ir.-Tur.

Chardinia orientalis (L.) Kuntze, "çağlaotu", 17, Quercus fields, 912 m., 14.05.2006, HA 5021, Ir.Tur.

Cichorium intybus L. "hindiba", 66, rocky slopes, 1000 m., 05.07.2007, MUÖ 2594a.

Cirsium arvense (L.) Scop. "köygöçüren", 63, roadsides, 1360 m., 14.09.2007, SYO 2974.

C. leucocephalum (Willd.) Spreng. subsp. penicillatum (K. Koch) Greuter, "üç hamurkesen", 64, rocky places, 1800 m, 25.09.2006, SYO 2847. Cousinia canescens DC. "kızan dikeni", 56, rocky places, 1800 m, 09.07.2006, SYO 2803, Ir-Tur.

Cota austriaca (Jacq.) Sch. Bip., "babuçça", 49, Quercus fields, 1200 m., 15.05.2006, HA 5196.

C. coelopoda (Boiss.) Boiss. var. bourgaei (Boiss.) Özbek \& Vural, "çiçekçi papatyası", 20, rocky slopes, 1270 m., 04.07.2007, MUÖ 2525.

C. tinctoria (L.) J. Gay ex Guss. var. tinctoria, "boyacı papatyası", 8, Quercus fields, 1225 m., 05.07.2007, MUÖ 2553.

Crepis foetida L. subsp. rhoeadifolia (M. Bieb.) elak, "sakarkanak", 4, roadsides, 1400 m., 13.04.2006, MV 9355.

C. sancta (L.) Bornm. "yaban kıskısı", 16, meadows, 1084 m, 15.05.2006, HA 5134.

Crupina crupinastrum (Moris) Vis. "gelindöndüren", 38, roadsides, rocky slopes, 1400-1640 m, 11.06.2006, MUÖ 2069.

Cyanus pichleri (Boiss.) Holub subsp. pichleri, "düğmeli ot", 33, high mountain steppe, 1885 m, 11.06.2006, MUÖ 2054.

Echinops spinosissimus Turra subsp. bithynicus (Boiss.) Greuter, "kirpibaşı", 64, rocky places, 1912 $m, 25.09 .06$, SYO 2848, Ir-Tur.

Filago arvensis L. "keçeotu", 28, rocky slopes, 1170 m., 02.07.2007, MUÖ 2450.

F. anatolica (Boiss. \& Heldr.) Chrtek \& Holub, "ana keçeotu", 33, rocky places, 1845 m., 05.07.2007, MUÖ 2565, Ir.-Tur.

Helichrysum arenarium (L.) Moench subsp. aucheri (Boiss.) P.H. Davis \& Kupicha, "yayla çiçeği", 61, stony slopes, 1280 m., 12.07.2005, MV 9168, End., LC, Ir.-Tur.

H. armenium DC. subsp. araxinum (Kirp.) Takht. "aras altınotu", 61, stony slopes, 1280 m., 
12.07.2005, MV 9163.

H. armenium DC. subsp. armenium, "altınotu", 45, stony slopes, 1150 m., 12.07.2005, MV 9154, Ir.-Tur.

H. plicatum DC. subsp. plicatum, "mantuvar", 39 high mountain steppe, 1885 m, 11.06.2006, MUÖ 2050.

Hieracium pannosum Boiss. "acıkanak", 22, rocky places, 1375 m., 04.07.2007, MUÖ 2551, E. Medit.

*Inula anatolica Boiss. "kaya andızı", 65, high mountain steppe, 2150 m., 07.07.2007, MUÖ 2597.

I. fragilis Boiss. \& Hausskn. "gevrek andızotu", 20, rocky slopes, 1270 m., 04.07.2007, MUÖ 2529, End., VU, Ir.-Tur.

I. salicina L. "su andızotu", 60 , edge of stream, 1500 m., 07.07.2007, MUÖ 2615, Euro-Sib.

Klasea cerinthifolia (Sm.) Greuter \& Wagenitz, "topbaş", 18, rocky slopes, 1170 m., 02.07.2007, MUÖ 2463.

K. oligocephala (DC.) Greuter \& Wagenitz, "saplı topbaş", 67, meadows and rocky places, $950 \mathrm{~m}$. 03.06.2007, HA 5582, Ir-Tur.

Lactuca mulgedioides (Vis. \& Pan ic) Boiss. \& Kotschy ex Boiss. "muş marulu",21, Quercus fields, 912 m., 14.05.2006, MUÖ 1969.

L. viminea (L.) J. Presl \& C. Presl, "çukurçıtlığı", 9, rocky slopes, 1580 m., 07.07.2007, MUÖ 2608. Lapsana communis L. subsp. intermedia (M. Bieb.) Hayek var. intermedia, "şebrek", 13, cultivated area, 900 m., MV 9530.

Leontodon crispus Vill. subsp. setulosus (Hal.) Kupicha, "aslandişi", 34, rocky places, 1630 m, HA 5454.

Picnomon acarna (L.) Cass. "kılçıkdiken", 30, rocky slopes, 940 m., 13.09.2007, SYO 2971, E. Medit.

Picris hieracioides L. subsp. hieracioides, "deli şiro", 44, roadsides, 1250 m, 06.07.2006, SYO 2695, Euro-Sib.

P. pauciflora Willd., "kum şirosu", 34, rocky places, 1670 m, 17.05.2006, HA 5316, E. Medit.

Pilosella hoppeana (Schult.) F.W. Schultz \& Sch. Bip. subsp. testimonialis (Naegli ex Peter) P.D. Sell \& C. West, "saplı tırnakotu", 33, high mountain steppe, 1875 m, 07.07.2006, SYO 2718.

Psephellus mucronifera (DC) Wagenitz, "tülübaş", 46, kayalık alanlar 1385 m, 11.06.2005, Kandemir 6954, End., LC, Ir.-Tur.

P. psephelloides (Freyn. \& Sint.) Wagenitz, "eğin tülübaşı", 51, kalker kaya çatlakları, 1180 m,
13.06.2004, Kandemir 6088, End., CR, Ir.-Tur.

P. pyrrhoblephanus (Boiss.) Wagenitz, "deli tülübaş", 60, edge of stream, rocky places, 1518 m, 05.06.2007, HA 5663 End., LC, Ir-Tur.

Pulicaria dysenterica (L.) Bernh. subsp. dysenterica, "yaraotu", 32, stony slopes, 1490 m, MV 9137.

Reichardia dichotoma (Vahl) Freyn, "karasakız", 4, serpentine stony slopes, $915 \mathrm{~m}, 13.07 .2005, \mathrm{MV}$ 9196, Ir-Tur.

Scorzonera inaequiscapa Boiss. "guzer", 40, steppe, 1565 m., 16.05.2006, HA 5276, End., LC. S. incisa DC. "kanık", 17, Quercus fields, 912 m., 14.05.2006, HA 5036, Ir-Tur.

S. latifolia (Fisch. \& C.A. Mey.) DC. var. latifolia, "dağsakızı", 66, rocky slopes, 1000 m., 05.07.2007, MUÖ 2594, Ir.-Tur.

S. mollis M. Bieb. subsp. mollis, "iskorçina", 53, steppe, 1500 m., 14.05.2006, HA 5066.

S. papposa DC. "tekecan", 49, Quercus fields, 1200 m., 15.05.2006, HA 5185, Ir.-Tur.

S. pseudolanata Grossh. "keçimemesi", 40, steppe, 1565 m., 16.05.2006, HA 5278, Ir.-Tur.

S. semicana DC. "kıvrım", 53, serpentine, 1500 m., 14.05.2006, HA 5066a, End., LC, Ir.-Tur.

S. tomentosa L. "alabent", 1, stony slopes, 1700 m., 13.07.2005, MV 9264, End., LC, Ir.-Tur.

Senecio rasemosus (M. Bieb.) DC. "şiro", 33, high mountain steppe, 1885 m., 11.06.2006, MUÖ 2066, Ir-Tur.

S. vernalis Waldst. \& Kit. "kanarya otu", 33, high mountain steppe, 1885 m., 11.06.2006, MUÖ 2065. Tanacetum argenteum (Lam.) Willd. subsp. canum (K. Koch) Grierson var. canum, "bodur pireotu", 66, limestone rock cracks, 1285 m, 13.06.2004, Kandemir 6091.

T. armenum (DC.) Sch. Bip., "kaya papatyası", 33, rocky places, 1900 m., 02.06.2007, HA 5499.

T. densum (Labill) Sch. Bip. subsp. eginense Heywood, "fırat pireotu", 46, rocky places, 1663 m, 11.06.2005, HA 5354, End., NT.

T. zahlbruckneri (Náb.) Grierson, “özge pireotu”, 6, roadsides, rocky slopes, 1320-1690 m, 11.06.2006, MUÖ 2078, End., LC, Ir-Tur.

Taraxacum buttleri Soest, "karahindiba", 34, rocky places, 1670 m., 17.05.2006, HA 5315.

T. microcephaloides Soest, "ebemçıtlığı", 64, meadows, 1795 m., 25.09.2006, SYO 2846. End., LC, Ir.-Tur.

T. montanum (C.A. Mey.) DC. "dağ hindibası", 2, 
steppe, 1680 m, 24.09.2006, SYO 2836, Ir.-Tur. T. scaturiginosum G.E. Haglund, "kıvırkıvır", 61, rocky slopes, 1300 m., 14.04.2006, MV 9388.

T. serotinum (Waldst. \& Kit.) Fisch. 38, roadsides, rocky slopes, 950 m., 15.09.2007, SYO 2984.

T. syriacum Boiss. "çöl çıtlığı", 1, stony slopes, 1600 m., 13.07.2005, MV 9254, Ir.-Tur.

Tragopogon porrifolius L. subsp. Iongirostris (Sch. Bip.) Greuter, "helevan", 40, rocky places, 870 m., 15.05.2006, HA 5148.

T. pterocarpus DC. "boz yemlik", 25, serpentine, 1600 m., 03.06.2007, HA 5529, Ir-Tur.

Tripleurospremum oreades (Boiss.) Rech. f. var. oreades, "hoşhoş", 31, meadows, 1200 m. 14.04.2006, MV 9385.

T. sevanense (Manden.) Pobed. "hanım gödesi", 33, high mountain steppe, 1885 m., 11.06.2006, MUÖ 2051.

Xeranthemum annuum L. "kağıtçiçeği", 32, stony slopes, 1190 m., 12.07.2005, MV 9114.

BERBERIDACEAE / KARAMUKGILLER

Berberis crataegina DC. "karamuk", 55, Quercus fields, 1287-1313 m., 14.05.2006, HA 5074.

Leontice leontopetalum L. "kırbaş", 6, roadsides, 1400 m., 13.04.2006, MV 9349.

\section{BORAGINACEAE / HODANGILLER}

Alkanna hirsutissima (Bertol) A. DC. "tüylü havacıva", 28, rocky slopes, 1170 m., 02.07.2007, MUÖ 2478, Ir.-Tur.

A. megacarpa A. DC. "yamaç havacıvası", 6, roadsides, rocky slopes, 1320-1690 m, 11.06.2006, MUÖ 2095, End., LC, Ir.-Tur.

Anchusa azurea Mill. var. azurea, "sığırdili", 12, roadsides, 08.07.2006, SYO 2761.

A. leptophylla Roem. \& Schult. subsp. leptophylla, "ballık", 29, rocky slopes, 1500 m., 02.07.2007, MUÖ 2503.

Asperugo procumbens L. "nevazilotu", 55, Quercus fields, 1287-1313 m, 14.05.2006, HA 5072, Euro-Sib.

Brunnera orientalis I.M. Johnst. "minik göğce", 13, meadows, 900 m, 15.04.2006, MV 9397, EuroSib.

Cerinthe minor L. subsp. auriculata (Ten.) Domac, "livarotu", 44, roadsides, 1252 m, 07.07.2006, SYO 2699.

Echium italicum L. "kurtkuyruğu", 44, roadsides, 1252 m, 07.07.2006, SYO 2691, E. Medit.

Heliotropium europaeum L. "akrep otu", 3, roadsides, 960 m, 24.09.06, SYO 2833, Ir.-Tur.
Macrotomia densiflora (Ledeb.) McBride, "kocaeğnik", 6, rocky slopes, 1686 m, 08.07.2006, SYO 2783, Ir.-Tur.

Moltkia coerulea (Willd.) Lehm. "mavi kesen", 55, steppe, 1287-1313 m, 14.05.2006, HA 5121, Ir.-Tur.

Myosotis alpestris F. W. Schmidt subsp. alpestris, "boncukotu", 34, rocky places, 1670 m, 17.05.2006, HA 5314.

M. lithospermifolia Hornem. "taş boncukutu", 55, Quercus fields, 1287-1313 m, 14.05.2006, HA 5089.

M. sylvatica Hoffm. subsp. rivularis Vestergr. "keleş unutmabeni", 33, meadows, 1870 m, 15.05.2006, HA 5312.

Nonea stenosolen Boiss. \& Balansa, "sormukotu", 33, calcareous rocky places, $1850 \mathrm{~m}, 17.05 .2006$, HA 5331, End., LC, Ir.-Tur.

Onosma affinis Hausskn. ex Riedl, "kardeş emziği", 61, slightly inclined slopes, 1685 m, 13.06.2013, Kandemir 10392, End., EN, Ir.-Tur.

0. alborosea Fisch. \& C.A. Mey. subsp. alborosea var. alborosea, "kaya emceği", 46, rocky places, 1230 m., 05.07.2007, MUÖ 2578, Ir.-Tur.

*0. aff. caerulescens Boiss. "şincar", 33, rocky places, $1875 \mathrm{~m}, 07.07 .2006$, SYO 2720, Ir.-Tur.

O. discedens Hausskn. ex Bornm. "fırat emceği", 23, serpentine, $1502 \mathrm{~m}, 12.06 .2004$, Kandemir 6085, End., VU, Ir.-Tur.

O. mollis DC. "divanköşk", 68, 1573 m, 05.07.2005, Kandemir 7029, Ir.-Tur.

*o. aff. obtusifolia Hauuskn. \& Sint. ex Rindl. "mera şincarı", 25, serpentine stony slopes, 1400 m, 13.07.2005, MV 9207, End., EN, Ir.-Tur.

*0. rechingeri Riedl. "geç mijmijok", 46, 1385 m, 11.06.2005, Kandemir 6957, End., LC, Ir.-Tur.

0. sericea Willd. "kağıt emcek", 28 , rocky slopes, 1170 m., 02.07.2007, MUÖ 2462, Ir.-Tur.

o. taurica Willd. var. taurica, "emzikotu", 41 , Juniperus and Quercus mixed area, $1530 \mathrm{~m}$, 16.05.2006, HA 5265.

Paracaryum cappadocicum Boiss. \& Balansa, "peri çarşağı", 10, under marbled stony slopes, 1582 m., 12.7.2005, ADK 2461, End., LC, Ir.-Tur.

P. cristatum (Schreb.) Boiss. subsp. cristatum, "ana çarşakotu", 10, stony slopes, Helichrysum, Papaver arasında, 12.7.2005, ADK 2462, End., LC, Ir.-Tur.

P. sintenisii Hausskn. ex Bornm. "eğin çarşağı", 25, serpentine, 1354 m, 12.7.2005, ADK 2459, Ir.Tur. 
Phyllocara aucheri (A.DC.) Guşul. "karadindik", 13, meadows, $900 \mathrm{~m}, 15.04 .2006$, MV 9486.

Rindera lanata (Lam.) Bunge var. lanata, "yünlü gelin", 2, rocky places, $1686 \mathrm{~m}, 08.07 .2006$, SYO 2790.

\section{BRASSICACEAE / TURPGILLER}

Aethionema arabicum (L.) Andrz. ex DC. "arap taşçantası", 39, rocky places, 870 m., 15.05.2006, HA 5144.

Ae. armenum Boiss. "taşçantası", 41, Juniperus and Quercus mixed area, 1030 m., 16.05.2006, HA 5255, Ir.-Tur.

Ae. capitatum Boiss. \& Balansa, "başmürselotu", 25, serpentine stony slopes, 1460 m., 13.07.2005, MV 9228, End., NT.

Ae. grandiflorum Boiss. \& Hohen var. grandiflorum, "koca kayagülü", 29, rocky slopes, 1500 m., 02.07.2007, MUÖ 2500, Ir.-Tur.

Ae. iberideum (Boiss.) Boiss. "ak kayagülü", 33 rocky places, 1900 m., 02.06.2007, HA 5502.

Ae. oppositifolium (Pers.) Hedge, "bodur kayagülü", 53, serpentine, 1500 m., 14.05.2006, HA 5064.

Ae. ozbekii Yıld. "özbek kayagülü", 33, steppe, 1885 m., 11.06.2006, MUÖ 2061, End., EN, Ir.-Tur.

Ae. spicatum Post, "gül taşçantası", 33, calcareous rocky places, 1870 m, 07.07.2006, SYO 2706.

Alliaria petiolata (M. Bieb.) Cavara \& Grande, "sarımsak hardalı", 61, rocky slopes, 1300 m., 14.04.2006, MV 9388.

Alyssum alyssoides (L.) L. "deliotu", 13, cultivated area, 900 m., 15.04.2006, MV 9543.

A. armenum Boiss. "ağrı kevkesi", 33, rocky slopes, 1875 m, 07.07.2006, SYO 2726b.

A. condensatum Boiss. \& Hausskn. subsp. flexibile (Nyár.) T.R. Dudley, "gevrek kuduzotu", 33, high mountain steppe, 1885 m., 11.06.2006, MUÖ 2056.

A. filiforme Nyar. "telli kevke", 67, meadows and rocky places, 950 m., 03.06.2007, HA 5573, End., LC, Ir.-Tur.

A. macropodum Boiss. \& Balansa var. macropodum, "saplı kevke", 57, rocky places, 1800 m., 08.06.2007, HA 5612, End., LC, Ir.-Tur.

A. pateri Nyár subsp. pateri, "kanatlı kevke", 69, rocky slopes, 860 m., 03.07.2007, MUÖ 2508, End., LC, Ir.-Tur.

A. sibiricum Willd. "kedidili", 23, serpentine, 1500 m., 20.04.2004, Kandemir 6862.
A. strigosum Banks \& Sol. subsp. strigosum, "dökük kuduzotu", 17, Quercus fields, 912 m., 14.05.2006, HA 5052.

A. szovitsianum Fisch. \& C.A. Mey. "çar kuduzotu", 55, Quercus fields, 1287-1313 m., 14.05.2006, HA 5101.

*A. trichostachyum Rupr, "sülün kevke", 70, meadows, 2238 m., 04.06.2007, HA 5647.

A. xanthocarpum Boiss. "dalsız kevke", 53, serpentine, 1500 m., 14.05.2006, HA 5065.

Arabis alpina L. subsp. alpina, "kaz teresi", 11, rocky slopes, 1150 m., HA 5206.

A. nova Vill. "tıfıl kazteresi", 4, roadsides, 1400 m., 13.04.2006, MV 9356.

Barbarea auriculata Hausskn. ex Bornm. var. auriculata, "kulaklı nicarotu", 46, 1589 m., 11.06.2005, Kandemir 6961, End., CR.

B. plantaginea DC. "götlezgötü", 5, rocky places, 1343 m., 06.06.2007, HA 5669.

Brassica elongata Ehrh. "uzun şalgam", 69, rocky slopes, 860 m., 03.07.2007, MUÖ 2519, Ir.-Tur.

Camelina laxa C. A. Mey. "eğri ketentere", 66, rocky slopes, 1000 m., 05.07.2007, MUÖ 2590.

C.rumelica Velen, "ketentere", 71, meadows, 1520 m., 02.06.2007, HA 5508.

Capsella bursa-pastoris (L.) Medik. "çobançantası", 55, Quercus fields, 1287-1313 m., 14.05.2006, HA 5073.

Choriospora tenella (Pall.) DC. "kokar külünk", 33 rocky places, 1900 m., 02.06.2007, HA 5493.

Clypeola aspera (Grauer) Turrill, "kıllı akçeotu", 33, rocky places, 1630 m., 02.06.2007, HA 5446, Ir.-Tur.

C. jonthlaspi L. "akçeotu", 52, rocky places, 890 m., 04.07.2007, MUÖ 2562.

Coluteocarpus vesicaria (L.) Holmboe subsp. vesicaria, "patarık otu", 42, Quercus fields, 1530 m., 16.05.2006, HA 5263, Ir.-Tur.

Conringia clavata Boiss. "topuztelkari", 55, Quercus fields, 1287-1313 m., 14.05.2006, HA 5111. C. planisiliqua Fisch. \& C.A. Mey. "telkariotu", 25, serpentine, 1600 m., 03.06.2007, HA 5548, Ir.-Tur. Draba bruniifolia Steven subsp. bruniifolia, "kaya dolaması", 71, slopes, 1520 m., 02.06.2007, HA 5525.

D. nuda (Bélanger) Al-Shahbaz \& M. Koch, "Cıbıl dolama", 6, rocky slopes, 1481 m., 16.05.2006, HA 5286, Ir.-Tur.

D. rigida Willd. var. rigida, "diri dolama", 11, rocky slopes, 1150 m., 15.05.2006, HA 5207. 
*Erysimum leucanthemum (Stephan ex Willd.) B. Fedtsch. "bayır zarifesi", 25, serpentine, 1600 m., 03.06.2007, HA 5536.

E. pycnophyllum J. Gay, "yamaç zarifesi", 53, serpentine, 1500 m., 14.05.2006, HA 5068, Ir.-Tur. E. sintenisianum Bornm. "köşeli zarifeotu", 70, meadows, 2238 m., 04.06.2007, HA 5646, End., LC, Ir.-Tur.

E. smyrnaeum Boiss. \& Balansa, "zeybek zarifesi", 69, rocky slopes, 860 m., 03.07.2007, MUÖ 2511.

E. uncinatifolium Boiss. "dadaş zarifeotu", 70, meadows, 2238 m., 04.06.2007, HA 5646a, Euro-Sib.

Fibigia clypeata (L.) Medik. var. eriocarpa (DC.) Post, "sikkeotu", 14, roadsides, 1270 m. 14.05.2006, MV 9540.

F. macrocarpa (Boiss.) Boiss. "koca sikkeotu", 61, stony slopes, 1280 m., 12.07.2005, MV 9173.

Hesperis bicuspidata (Willd.) Poir, "gecemenekşesi", 34, rocky places, 1630 m., 02.06.2007, HA 5443, End., LC, Ir.-Tur.

H. thyrsoidea Boiss. "salkım akşamyıldızı", 43, rocky places, 1400 m., 15.05.2006, HA 5231, Ir.Tur.

*Isatis kotschyana Boiss. \& Hohen. "cilo çivitotu", 4, roadsides, 1400 m., 13.04.2006, MV 9347, Ir.Tur.

I. undulata Aucher ex Boiss. "etekli çivitotu", 28, rocky slopes, 1400 m, 02.07.2007, MUÖ 2486, End., EN, Ir.-Tur.

Iberis simplex DC. "civanotu", 53, serpentine stony slopes, 1500 m., 14.05.2006, HA 5063.

Lepidium draba L. "diğnik", 14, roadsides, 1270 m., 14.05.2006, MV 9545.

Microthlaspi perfoliatum (L.) F.K. Mey. "giyle", 13, meadows, 900 m., 15.04.2006, MV 9392.

Myagrum perfoliatum L. "üçodaotu", 69, rocky slopes, 860 m., 03.07.2007, MUÖ 2512.

Nasturtium officinale R. Br. "suteresi", 33, meadows, 1900 m., 06.06.2007, HA 5678.

Neslia paniculata (L.) Desv. subsp. thracica (Velen.) Bornm. "göçmen hardalı", 17, Quercus fields, 912 m., 14.05.2006, HA 5054.

Physocardamum angustifolium (Hausskn. ex Bornm.) Kandemir, "seyyah otu", 34, rocky places and slopes, 1670 m., 17.05.2006, End., CR, Ir.-Tur. *Physoptychis haussknechtii Bornm., "gezertere", 25, serpentine stony slopes, 1460 m., 13.07.2005, MV 9223, End., EN, Ir.-Tur.

Raphanus raphanistrum L. subsp. raphanistrum, "eşek turpu", 17, Quercus fields, 912 m., 14.05.2006, HA 5053.

Rapistrum rugosum (L.) All. "kedi turpu", 69, rocky slopes, 860 m., 03.07.2007, MUÖ 2509.

Ricotia aucheri (Boiss.) B. L. Burtt, "boz cavlak", 16, meadows, 1084 m., 15.05.2006, HA 5136, Ir.Tur.

Sisymbrium orientale L. "tarla bülbülotu", 12, roadsides, 08.07.2006, SYO 2756.

Sobolewskia clavata (Boiss.) Fenzl, "akyelotu", 50, rocky places, 980 m., 16.05.2006, HA 5307, Ir.-Tur.

Tchihatchewia isaditea Boiss. "allıgelin", 53, serpentine, 1500 m., 14.05.2006, HA 5067, End., VU, Ir.-Tur.

\section{CAMPANULACEAE / ÇANÇiçEĞiGiLLER}

Asyneuma amplexicaule (Willd.) Hand.Mazz. subsp. amplexicaule var. amplexicaule, "hoşdeğnek", 33, rocky places, 1875 m., SYO 2717.

A. limonifolium (L.) Janch. subsp. limonifolium, "tavşanekmeği", 6, roadsides, rocky slopes, 1500 m., 11.06.2006, MUÖ 2087.

A. rigidum (Willd.) Grossh. subsp. rigidum, "nujdan", 37, steppe, 1671 m., SYO 2742, Ir.-Tur.

A. virgatum (Labill.) Bornm. subsp. virgatum, "çiçeklideğnek", 28, rocky slopes, 1170 m., 02.07.2007, MUÖ 2489.

Campanula conferta A. DC. Ir.-Tur. "saklı çançiçeği", 33, steppe, 1885 m., 11.06.2006, MUÖ 2048.

C. ptarmicifolia Lam. "ters çançiçeği", 74, yol kenarı, 1226 m., 13.06.2004, Kandemir 6099, End., VU, Ir.-Tur.

C. rapunculoides L. "elmacık", 12, roadsides, 08.07.2006., SYO 2762, Euro-Sib.

C. strigillosa Boiss. "peri çıngırağı", 25, stony slopes, serpentine, 1460 m., 13.07.2006, MV 9224, End., LC, E. Medit.

C. strigosa Banks \& Sol. "kıraç çanı", 4, rocky slopes, 1040 m., HA 5300.

C. yildirimlii Kit Tan \& Sorger, "yiğit çıngırağı", 61, stony slopes, 960 m., 14.09.2007, SYO 2980, End., EN.

CANNABACEAE / KENEVIRGILLER

Celtis planchoniana K.I.Chr. "dahum", 4, serpentine stony slopes, 915m., 13.07.2005, MV 9201.

C. tournefortii Lam. "dardağan", 27, cultivated area, 1143 m., 02.07.2007, MK 1968.

CAPRIFOLIACEAE / HANIMELIGILLER 
Centranthus longiflorus Steven subsp. longiflorus, "mahmuzçiçeği", 39, rocky places, 870 m., 15.05.2006, HA 5153, Ir.-Tur.

Cephalaria speciosa Boiss. \& Kotschy, "yıldız pelemiri", 63, rocky slopes, 1360 m., 14.09.2007, SYO 2972, End., LC, Ir-Tur.

Lonicera etrusca Santi var. etrusca, "dokuzdon", 12, Quercus fields, 08.07.2006., SYO 2751, E. Medit.

L. nummularifolia Jaub. \& Spach subsp. nummularifolia, "tavşançili", 28, rocky slopes, 1170 m., 02.07.2007, MUÖ 2492.

Morina persica L. var. persica, "merdivençiçeği", 66, rocky slopes, 1000 m., 05.07.2007, MUÖ 2590a, Ir.-Tur.

Pterocephalus pinardii Boiss. "yurt cücükotu", 25, serpentine stony slopes, 1400 m., 13.07.2005, MV 9220, End., LC, E. Medit.

Scabiosa columbularia L. subsp. columbularia, "uyuzotu", 67, rocky places, 950 m., 03.06.2007, HA 5585.

S. crinita Kotschy \& Boiss. "sarıpuk", 61, stony slopes, 1280 m., 12.07.2005, MV 9165, Ir.-Tur.

S. rotata M. Bieb., "top uyuzotu", 28, rocky slopes, 1400 m., 02.07.2007, MUÖ 2459, Ir.-Tur.

Valeriana dioscoridis Sm. "çobanzurnası", 14, roadsides, 1270 m., 14.05.2006, MV 9566, E. Medit.

V. officinalis L. "kediotu", 17, Quercus fields, 912 m., 14.05.2006, HA 5034.

Valerianella coronata (L.) DC. "taçlı kuzugevreği", 55, Quercus fields, 1287-1313 m., 14.05.2006, HA 5086.

V. dactylophylla Boiss. \& Hohen. "el kuzugevreği", 39, rocky places, 870 m., 15.05.2006, HA 5158, Ir. Tur.

V. pumila (L.) DC. "bağ kuzugevreği", 34, rocky places, 1630 m., 02.06.2007, HA 5479.

V. vesicaria (L.) Moench, "kuzugevreği", 17, Quercus fields, 912 m., 14.05.2006, HA 5043.

\section{CARYOPHYLLACEAE / KARANFILGILLER}

*Arenaria antitaurica McNeill. "kaya kumotu", 55 rocky places, 1715 m., 09.07.2006., SYO 2800, End., LC.

A. kotschyana Fenzl subsp. kotscyhana, "niğde kumotu", 33, rocky places, 1875 m, 07.07.2006, SYO 2726, End., LC.

A. serpyllifolia L. subsp. leptoclados (Rchb.) Nyman. "kuru kumotu", 49, Quercus fields, 1200 m., 15.05.2006, HA 5201.
Cerastium brachypetalum Pers. subsp. roeseri (Boiss. \& Heldr.) Nyman, "gevşek boynuzotu", 17, Quercus fields, 912 m., 14.05.2006, HA 5018, E. Medit.

C. dichotomum L. subsp. dichotomum, "çatal boynuzotu", 17, Quercus fields, 912 m., 14.05.2006, HA 5023.

C. dichotomum L. subsp. inflatum (Link) Cullen, "patlak boynuzotu", 16, meadows, 1084 m., 15.05.2006, HA 5132.

C. perfoliatum L. "ekin boynuzotu", 55, Quercus fields, 1300 m., 14.05.2006, HA 5070.

Eremogone ledebouriana (Fenzl) Ikonn. "iğne kumotu", 39, rocky places, 870 m, 15.05.2006, HA 5157, End., LC, Ir.-Tur.

*E. macrantha (Schischk.) Ikonn. "yamaç kumotu", 44, rocky places, 1400 m., 15.05.2006, HA 5222, Ir.-Tur.

*Dianthus carmelitarum Reut. ex Boiss. 48, "samsu", rocky slopes, 1025 m., 10.06.2006, MUÖ 2026, End., LC, Euro-Sib.

D. crinitus Sm. var. crinitus, "uzunçanak", 23, serpentine, 1467 m., 05.07.2005, Kandemir 7009. D. floribundus Boiss. "kırk karanfil", 8, Quercus fields, 1225 m., 05.07.2007, MUÖ 2589, Ir.-Tur.

D. masmenaeus Boiss. var. glabrescens Boiss. "etek karanfili", 44, rocky places, 1252 m., 06.07.2006, SYO 2696, End., LC, Ir.-Tur.

D. orientalis Adams, "yar karanfili", 48, rocky slopes, 1025 m., 10.06.2006, MUÖ 2042.

Minuartia juniperina (L.) Maire \& Petitm. "hanım şiltesi", 25, rocky slopes, 1170 m., 02.07.2007, MUÖ 2491.

M. hamata (Hausskn.) Mattf. "koruotu", 17, Quercus fields, 912 m., 14.05.2006, HA 5042.

Paronychia cataonica Chaudhri, "gürün etyaranı", 8, under Juniperus oxycedrus, 964 m., 15.05.2006, HA 5180, End., VU.

P. kemaliya Chaudri, "eğin etyaranı", 8, under Juniperus oxycedrus, 964 m., 15.05.2006, HA 5177, End., EN.

P. kurdica Boiss. subsp. kurdica var. kurdica, "boz kepekotu", 25, serpentine slopes, 1460 m., 13.07.2006, MV 9215.

Petrorhagia cretica (L.) P.W. Ball \& Heywood, "ada zarçiçeği", 21, rocky slopes, 1130 m., 04.07.2007, MUÖ 2545.

Saponaria prostrata Willd. subsp. anatolica Hedge, "ana sabunotu", 26, rocky slopes, 1380 m., 04.07.2007, MUÖ 2554, End., LC, Ir.-Tur. 
Silene ampullata Boiss. "hoş kıyışak", 44, rocky places, 1400 m., 15.05.2006, HA 5230, Ir.-Tur.

S. capitellata Boiss. "kavuklu nakıl", 34, rocky places, 1670 m., 17.05.2006, HA 5316, End., LC, Ir.-Tur.

S. compacta Fisch. ex Hornem. "kanlıbasıraotu", 21, rocky slopes, 1130 m., 04.07.2007, MUÖ 2543.

S. conoidea L. "Şıvananotu", 17, Quercus fields, 912 m., 14.05.2006, HA 5057.

S. laxa Boiss. \& Kotschy, "gevşek simotu", 4, rocky places, 1040 m., 16.05.2006, HA 5289, Ir.-Tur.

S. montbretiana Boiss. "fay nakılı", 11, rocky places, 1150 m, 15.05.2006, HA 5210, Ir.-Tur.

S. nerimaniae G.E. Genç, A. Kandemir \& I. Genç, "sultan nakılı", 24, serpentine slopes, 1502 m., 12.05.2005, i. Genç 481, End., VU, Ir.-Tur.

S. odontopetala Fenzl, "kunduzotu", 11, rocky places, 1150 m., 15.05.2006, HA 5219.

S. spergulifolia (Desf.) M.Bieb. "ana nakı", 3, roadsides, steppe, 1090 m., 12.06.2006, MUÖ 2100, Ir.-Tur.

*S. viscosa Pers. "konya gıvışkanı", 66, hareketli, taşlı yamaçlar, 1285 m, 05.07.2005, Kandemir 7039.

S. vulgaris (Moench) Garcke var. commutata (Guss.) Coode \& Cullen, "ecibübü", 17, Quercus fields, 912 m., 14.05.2006, HA 5019.

Telephium imperati L. subsp. orientale (Boiss.) Nyman, "zulzula", 33, stony slopes, 1490 m. 12.07.2005, MV 9133.

CELESTRACEAE / IĞAĞACIGILLER

Parnassia palustris L. "yürekyaprağı", 61, roadsides, 1000 m., 15.09.2007, SYO 2981.

\section{CISTACEAE / LADENGILLER}

Fumana procumbens (Dunal) Gren. \& Godr. "yer güneşotu", 25, serpentine, 1600 m., 03.06.2007, HA 5547.

Helianthemum nummularium (L.) Mill. subsp. nummularium, "güngülü", 56, rocky slopes, 12871313 m., 14.05.2006, HA 5118.

H. ledifolium (L.) Mill. "kuru güngülü", 72, 1046 m, 27.04.2005, Kandemir, 6872.

CLEOMACEAE / SAÇAKGÜLÜGILLER

Cleome ornithopodioides L. "taş saçakgülü", 25, stony slopes, serpentine, 1460 m., 13.07.2006, MV 9212, E. Medit.

\section{COLCHICACEAE / ACIÇIĞDEMGILLER}

Colchicum szovitsii Fisch. \& C.A. Mey. subsp. szovitsii, "katır çiğdemi", 34, rocky places, 1650 m., 14.04.2006, MV 9377, Ir.-Tur.

\section{CONVOLVULACEAE}

\section{TARLASARMAŞIĞIGILLER}

*Calystegia silvatica (Kit.) Griseb. "bürük", 3, edge of stream, 860 m., 03.07.2007, MK 1973.

Convolvulus arvensis L. "tarla sarmaşı̆̆ı", 33, steppe, 1885 m., 11.06.2006, MUÖ 2057.

C. galaticus Rost. ex Choisy, "boz sarmaşık", 38, roadsides, 1875 m., 07.07.2006, SYO 2732, Ir.-Tur. c. holosericeus M. Bieb. subsp. holosericeus, "gündüz sefası", 28, rocky slopes, 1200 m., 02.07.2007, MUÖ 2484.

C. pseudoscammonia K. Koch, "sarı yayılganı", 25, serpentine stony slopes, 1400 m., 13.07.2005, MV 9206, End., LC, Ir.-Tur.

*C. scammonia L. "bingözotu", 29, rocky slopes, 1500 m., 02.07.2007, MUÖ 2505, E. Medit.

Cuscuta palaestina Boiss. "arap cinsaçı", 62, roadsides, 950 m., SYO 2769.

\section{CORNACEAE / KIZILCIKGILLER}

Cornus sanguinea L. subsp. australis (C.A. Meyer) Jáv. "kansiğdiren", 13, cultivated area, 900 m., 15.04.2006, MV 9508, Euro-Sib.

\section{CRASSULACEAE / DAMKORUĞUGILLER}

Promethium sempervivoides (Fischer ex $M$. Bieb.) H. Ohba, "horozlelesi", 65, high mountain steppe, 2150 m., 07.07.2007, MUÖ 2597a.

*Rosularia libanotica (Strand ex L.) Samuelsson, "arap kayakoruğu", 38, rocky places, 1400-1640 m., 11.06.2006, MUÖ 2070, E. Medit.

* $\boldsymbol{R}$. radiciflora (Steud. ex Boiss.) Borissova subsp. glabra (Bornm.) Chamberlain \& Muirhead, "bodur kayakoruğu", 50, rocky places, 870 m., 16.05.2006, HA 5309, Ir.-Tur.

Sedum album L. "çoban kavurgası", 48, rocky slopes, 1025 m., 10.06.2006, MUÖ 2022.

*S. hispanicum L. "damkoruğu", 49, Quercus fields, 1200 m., 15.05.2006, HA 5193, Ir.-Tur.

S. pallidum M. Bieb. "koyunörmece", 8, under Juniperus oxycedrus, 964 m., 15.05.2006, HA 5176, Euro-Sib.

\section{CUCURBITACEAE / KABAKGILLER}

Bryonia multiflora Boiss. \& Heldr. "ülüngür", 44, rocky places, 1400 m., 15.05.2006, HA 5234, Ir.Tur.

\section{CYPERACEAE / HASIROTUGILLER}

Eleocharis acicularis (L.) Roem. \& Schult. var. acicularis, "cücesaz", 4, meadows, 900 m., 15.04.2006, MV 9335.

Scirpoides holoschoenus (L.) Sojak subsp. holoschoenus, "vurla", 14, damp area, 1270 m., 
14.05.2006, MV 9517.

\section{ELAEAGNACEAE / IĞDEGILLER}

Elaeagnus angustifolia L. var. angustifolia, "iğde", 14, roadsides, 1270 m., 14.05.2006, MV 9495.

\section{EUPHORBIACEAE / SÜTLEĞENGILLER}

Euphorbia cheiradenia Boiss. \& Hohen. "şirker", 33, steppe, 1885 m., 11.06.2006, MUÖ.2062, Ir.Tur.

E. condylocarpa M. Bieb. "gijiletri", 17, Quercus fields, 912 m., 14.05.2006, HA 5016.

E. denticulata Lam. "karasütlük", 34, rocky places, 1630 m., 02.06.2007, H.A 5447, Ir.-Tur.

\section{FABACEAE / BAKLAGILLER}

*Astragalus acicularis Bunge, "iğne geveni", 25 , serpentine, 1600 m., 03.06.2007, HA 5535, End. LC, Ir.-Tur.

A. anthylloides Lam. "torbalı geven", 42, steppe, 1468 m., 04.06.2007, HA 5637, Ir.-Tur.

A. asciocalyx Bunge, "külah geveni", 33, high mountain steppe, 1885 m., 11.06.2006, MUÖ 2055, Ir.-Tur.

A. cadmicus Boiss. "baba geveni", 34, rocky places, 1670 m., 17.05.2006, HA 5319, End., LC.

A. densifolius Lam. subsp. densifolius, "gümüş geven", 33, high mountain steppe, 1885 m., 11.06.2006, MUÖ 2052, Ir.-Tur.

A. elongatus Willd. subsp. elongatus, "yazı yoncası", 57, rocky places, 1800 m., 03.06.2007, HA 5623.

A. fragrans Willd., "mis geven", 33, meadows, 1900 m., 06.06.2007, HA 5682.

A. lineatus Lam. var. Iongidens (Freyn) Matthews, "patpat", 70, meadows, 2238 m., 04.06.2007, HA 5648, Ir.-Tur.

A. pendulus DC. "sırık geveni", 11, rocky slopes, 1150 m., 15.05.2006, HA 5216, Ir.-Tur.

A. pseudocylindraceus Boiss., "karasu geveni", 34, stony slopes, 1605 m, 13.06.2013, Kandemir 10395, End., CR, Ir.-Tur.

A. psoraloides Lam. "bayburt geveni", 23, serpentine, 1500 m., 10.06.2005, Kandemir 6845, Ir.-Tur.

A. tigridis Boiss. "dicle geveni", 56, rocky places, 14.05.2006, HA 5105, Ir.-Tur.

A. xylobasis Freyn \& Bornm. "kemaliye geveni", 4, rocky places, 1040 m., 16.05.2006, HA 5290, Ir.-Tur.

Cicer bijugum Rech. f. "pitrak nohut", 55, Quercus fields, 1287-1313 m., 14.05.2006, HA 5096, Ir.-Tur.
Colutea cilicica Boiss. \& Balansa, "patlangaç", 14, roadsides, damp area, 970 m., 14.05.2006, MV 9570.

Coronilla scorpioides (L.) W.D.J. Koch. "akrep burçağı", 8, under Juniperus oxycedrus, 964 m., 15.05.2006, HA 5161.

*Dorycinum graecum (L.) Ser. "ak kaplanotu", 58, 1550 m., 05.07.2005, Kandemir 7021, Euro-Sib.

D. pentaphyllum Scop. subsp. anatolicum (Boiss.) Gams, "kaplanotu", 14, roadsides, damp area, 970 m, 14.05.2006, MV 9479.

Ebenus haussknechtii Bornm. ex Hub.-Mor. "Harput morgeveni", 1, stony slopes, 1600 m., 13.08.2005, MV 9258, End., NT, Ir.-Tur.

E. laguroides Boiss. "morgeven", 19, rocky places, 850 m., 16.05.2006, HA 5311, End., LC, Ir.-Tur.

Genista aucheri Boiss. "bayır borcağı", 39, rocky places, 870 m., 15.05.2006, HA 5140, End., LC, Ir.-Tur.

G. albida Willd. "ak borcak", 28, rocky slopes, 1170 m., 02.07.2007, MUÖ 2474.

Hedysarum candidissimum Freyn, "hoş batalağı", 33, meadows, 1900 m., 06.06.2007, HA 5683, End., NT, Ir.-Tur.

H. pogonocarpum Boiss. "taş batalağı", 73, calcareous rocky places, 950 m., 10.06.2006, MUÖ.2020, End., LC.

H. varium Willd. subsp. varium, "batalak", 9, rocky slopes, 1580 m., 07.07.2007, MUÖ 2607.

Lathyrus brachypterus elak, "yemelik", 34, rocky places, 1670 m., 17.05.2006, HA 5312, End., LC.

L. sativus L. "mürdümük", 34, rocky places, 1630 m., 02.06.2007, HA 5464, E. Medit.

Lens culinaris Medik. subsp. orientalis (Boiss.) Ponert, "yasmık", 67, rocky places, 950 m., 03.06.2008, HA 5595.

Lotus corniculatus L. var. alpinus Ser. "gazalboynuzu", 31, meadows, 970 m., 15.09.2007, SYO 2983.

L. gebelia Vent. var. anthylloides Boiss. "gül gazalboynuzu", 73, calcareous rocky places, 950 m., 10.06.2006, MUÖ.2021, End., NT, Ir.-Tur.

Medicago coronata (L.) Bartol, "gevşek yonca", 17, Quercus fields, 912 m., 14.05.2006, HA 5039, E. Medit.

M. crassipes (Boiss.) E. Small, "hançer yoncası", 4, under Juniperus oxycedrus, 964 m., 15.05.2006, HA 5173 Ir.-Tur.

M. minima (L.) Bartol var. minima, "gurnik", 69, 
stony slopes, 860 m., 03.07.2007, MUÖ 2513.

M. monantha (C.A. Mey.) Trautv. "dağ gurniği", 25, serpentine, 1600 m., 03.06.2007, HA 5558a, Ir.-Tur.

M. monspeliaca (L.) Trautv. "bayır gurniği", 67, rocky places, 950 m., 03.06.2007, HA 5592, E. Medit.

M. radiata L. "hilal yonca", 4, under Juniperus oxycedrus, 964 m., 15.05.2006, HA 5167, Ir.-Tur.

M. sativa L. subsp. sativa, "karayonca", 28 , rocky slopes, 1170 m., 02.07.2007, MUÖ 2469.

M. rigidula (L.) All. var. rigidula, "kaba yonca", 25, serpentine, 1600 m., 03.06.2007, HA 5558.

Melilotus albus Desr. "ak taşyoncası", 52, in the valley, rocky places, 850 m., 06.07.2007, MUÖ 2595.

M. officinalis (L.) Desr. "kokulu yonca", 14, roadsides, damp area, 900 m., 14.05.2006, MV 9520.

Onobrychis cornuta (L.) Desv. "kuşkaçıran", 33, meadows, 1600 m., 02.06.2007, HA 5486, Ir.-Tur. o. galegifolia Boiss. "darp korungası", 4, serpentine slopes, 915 m., 13.07.2005, MV 9198, Ir.-Tur.

0. oxyodonta Boiss. var. armena (Boiss. \& Huet.) Aktoklu, "kır korungası", 6, rocky slopes, 13201690 m., 11.06.2006, MUÖ 2086.

o. oxyodonta Boiss. var. oxyodonta, "kır korungası", 34, rocky places, 1630 m., 02.06.2007, HA 5436.

Ononis spinosa L. subsp. leiosperma (Boiss.) irj. "demirdelen", 33, high mountain steppe, $1875 \mathrm{~m}$. 07.07.2006, SYO 2738.

Pisum sativum L. subsp. elatius (M. Bieb.) Aschers. \& Graebn. var. elatius, "boylu bezelye", 17, Quercus fields, 912 m., 14.05.2006, HA 5037.

Robinia pseudoacacia L. "yalancı akasya", 14, roadsides, damp area, 900 m., 14.05.2006, MV 9553.

*Securigera cretica (L.) Lassen, "ada körügeni", 42, steppe, 1468 m., 04.06.2007, HA 5639, E. Medit.

S. orientalis (Mill.) Lassen subsp. orientalis, "ala körügen", 14, roadsides, damp area, 970 m., 14.05.2006, MV 9490.

Trifolium campestre subsp. campestre Schreb. "üçgül", 13, meadows, 900 m., 15.04.2006, MV 9513.

T. pratense L. var. pratense, "çayır üçgülü", 31, meadows, 1200 m., 14.04.2006, MV 9391.
T. purpureum Lois. var. purpureum, "mor üçgül", 17, Quercus fields, 912 m., 14.05.2006, HA 5046.

Trigonella spicata Sibth. \& Sm. "başak boyotu", 56, roadsides, 1794 m., 05.06.2007, HA 5653.

T. spruneriana Boiss. "koç boynuzotu", 13, meadows, 900 m., 15.04.2006, MV 9549.

T. strangulata Boiss. "düğmeli boyotu", 67, meadows and rocky places, 950 m., 03.06.2007, HA 5589.

T. velutina Boiss., "ipek boyotu", 8, under Juniperus oxycedrus, 964 m., 15.05.2006, HA 5165.

Vicia cracca L. subsp. stenophylla Vel. "meşe fiği", 39, rocky places, 870 m., 15.05.2006, HA 5141.

V. lathyroides L. "çamfiği", 13, meadows, 900 m., 15.04.2006, MV 9539.

V. sativa L. subsp. sativa, "fiğ", 8 , under Juniperus oxycedrus, 964 m., 15.05.2006, HA 5040.

V. sericocarpa Fenzl var. sericocarpa, "çitfiği", 8, under Juniperus oxycedrus, 964 m., 15.05.2006, HA 5048.

\section{FAGACEAE / KAYINGILLER}

Quercus brantii Lindl. "karameşe", 39, rocky places, 870 m., 15.05.2006, HA 5155, Ir.-Tur.

Q. cerris L. "saçlımeşe", 15, Quercus fields, 1100 m., 23.09.06, SYO 2810, E. Medit.

Q. infectoria Oliv. subsp. veneris (A. Kern.) Meikle, "zindiyen", 15, Quercus fields, 1200 m., 23.09.06, SYO 2811.

Q. libani Oliv. "Iübnan meşesi", 39, rocky places, 870 m., 15.05.2006, HA 5159, Ir.-Tur.

Q. pubescens Willd. "tüylü meşe", 15, Quercus fields, 1100 m., 23.09.06, SYO 2816.

\section{GENTIANACEAE / GENTIYANGILLER}

Centaurium erytraea Rafn. subsp. turcicum (Velen.) Melderis, "tukulotu", 52, inner valley, rocky places, 850 m., 06.07.2007, MUÖ 2596.

Gentiana olivieri Griseb. "afat", 71, meadows, 1520 m., 02.06.2007, HA 5509, Ir.-Tur.

GERANIACEAE / TURNAGAGASIGILLER

Geranium purpureum Vill. "ebedön", 52, rocky places, 890 m., 06.07.2007, MUÖ 2563.

G. pyreanicum Burnm. f. "gelinçarşafı", 14, roadsides, 1270 m., 14.05.2006, MV 9536.

G. tuberosum L. "çakmuz", 18, rocky places, 915 m., 13.04.2006, MV 9338, Ir.-Tur.

HYPERICACEAE / KANTARONGILLER

Hypericum perforatum L. subsp. perforatum, 
"kantaron", 30, steppe, 1031m., 30.5, 06.07.2006, SYO 2681.

H. peshmenii Yıld., "şah lantaronu", 79, side of mill, limestone rocky crevices, 1100 m., 20.08.2013, Kandemir 10473, End., CR, Ir.-Tur.

H. pseudolaeve N. Robson, "keşiş kantaronu", 8 Quercus fields, 1225 m., 05.07.2007, MUÖ 2581, Ir.-Tur.

H. scabrum L. "karahasançayı", 39, rocky places, 870 m., 15.05.2006, HA 5146, Ir.-Tur.

H. thymopsis Boiss. "darende kantaronu", 25, stony slopes, 1460 m., 13.07.2005, MV 9219, End., NT, Ir.-Tur.

\section{IRIDACEAE / SÜSENGILLER}

Crocus biflorus Mill. subsp. tauri (Maw) B. Mathew, "berfan", 6, rocky places, 1680 m., 13.04.2006, MV 9343, Ir.-Tur.

C. kotschyanus K. Koch subsp. cappadocicus B. Mathew, "peri çiğdemi", 64, meadows, 1912 m. 25.09.2006, SYO 2845, End., NT, Ir.-Tur.

C. pallasii Goldb. subsp. pallasii, "güzçimi", 34 rocky places, 1630 m., 23.09.2006, SYO 2822.

Gladiolus atroviolaceus Boiss. "kıraç süseni", 34 rocky places, 1630 m., 02.06.2007, HA 5440, Ir. Tur.

G. italicus Mill. "kılıçotu", 13, meadows, 900 m., 15.04.2006, MV 9516.

G. kotschyanus Boiss. "çayır kılıçotu", 25, serpentine slopes, 1400 m., 13.07.2005, MV 9204 Ir.-Tur.

Iris caucasica Hoffm. subsp. turcica B. Mathew, "türk navruzu", 33, calcareous rocky places, 1850 m., 17.05.2006, HA 5324, Ir.-Tur.

I. persica L. "buzala", 6, roadsides, 1400 m., 13.04.2006, MV 9348, Ir.-Tur.

I. reticulata M.Bieb. var. reticulata, "karakörpeze", 34, rocky places, 1650 m., 14.04.2006, MV 9380, Ir.-Tur.

I. sari Schott ex Baker, "ana kurtkulağı", 55, steppe, 1300 m., 14.05.2006, HA 5092, End., LC, Ir.-Tur.

IXIOLIRIACEAE / KÖPEKOTUGILLER

*Ixiolirion tataricum (Pall.) Schult. \& Schult.f. var. tataricum, "köpekotu", 13, meadows, 900 m. 15.04.2006, MV 9571, Ir.-Tur.

JUGLANDACEAE / CEVIZGILLER

Juglans regia L. "ceviz", 13, cultivated area, 900 m., 15.04.2006, MV 9497.

JUNCACEAE / KOFAGILLER

Juncus articulatus L. subsp. articulatus, "camışotu", 69, stony slopes, 860 m., 03.07.2007, MUÖ 2518, Euro-Sib.

\section{LAMIACEAE / BALLIBABAGILLER}

Ajuga chamaepitys (L.) Schreb. subsp. chia (Schreber) Arcangeli var. chia, "acıgıcı", 55, Quercus fields, 1287-1313 m., 14.05.2006, HA 5097.

Clinopodium serypyllifolium (M. Bieb.) Kuntze subsp. brachycalyx (P.H. Davis) Brauchler, "şarşarçayı", 51, limestone rock cracks, 1570 m., 05.07.2005, Kandemir 7027, E. Medit.

Cyclotrichium niveum (Boiss.) Manden \& Scheng. "külotu", 45, rocky places, 1150 m., 12.07.2005, MV 9161, End., VU, Ir.-Tur.

Eremostachys moluccelloides Bunge, "benli sultan", 61, stony slopes, 1280 m., 12.07.2005, MV 9174, Ir.-Tur.

Lamium album L. subsp. crinitum (Montbret \& Aucher ex Benth.) Mennema, "kovanlık", 33, meadows, 1600 m., 02.06.2007, HA 5487, EuroSib.

L. amplexicaule L. var. amplexicaule, "baltutan", 38, rocky slopes, 1650 m., 14.04.2006, MV 9368. L. garganicum L. subsp. striatum (Sm.) Hayek var. striatum, "tel balıcak", 33, meadows and rocky places, 1600 m., 02.06.2007, HA 5487.

L. orientale (Fisch. \& C.A. Mey.) E.H.L. Krause, "güzelce", 17, Quercus fields, 912 m., 14.05.2006, HA 5022, Ir.-Tur.

L. purpureum L. var. purpureum, "ballıbaba", 13, meadows, 900 m., 15.04.2006, MV 9393., EuroSib.

Marrubium astracanicum Jacq. subsp. astracanicum, "mor yayotu", 33, high mountain steppe, 1885 m., 11.06.2006, MUÖ 2063.

M. parviflorum Fisch. \& C.A. Mey. subsp. oligodon (Boiss.) Seybold, "küllü bozotu", 74, roadsides, 1794 m., 05.06.2007, HA 5659, End., LC.

Mentha spicata L. subsp. spicata, "eşek nanesi", 12, edge of streams, 08.07.2006, SYO 2759.

Micromeria cremnophila Boiss. \& Heldr. subsp. anatolica P.H. Davis, "taş boğumcuğu", 46, rocky places, 1230 m., 05.07.2007, MUÖ 2579, End., LC, Ir.-Tur.

*M. elliptica K. Koch, "kaya yarpuzu", 32, stony slopes, 1490 m., 12.07.2006, MV 9142, Euro-Sib.

M. myrtifolia Boiss. \& Hohen, "boğumlu çay", 25, stony slopes, 1460 m., 13.07.2006, MV 9242.

Nepeta italica L. "eşekçayı", 32, stony slopes, 1190 m., 12.07.2006, MV 9122, E. Medit. 
N. nuda L. subsp. albiflora (Boiss.) Gams, "karaküncü", 32, stony slopes, 1190 m., 12.07.2006, MV 9108.

Origanum aff. acutidens (Hand.-Mazz) letsw. "zemul", 25, stony slopes, 1460 m., 13.07.2006, MV 9237, End., LC, Ir.-Tur.

o. vulgare L. subsp. gracile (K. Koch) letsw. "kuş zemulu", 62, rocky places, 1100 m, 08.07.2006, SYO 2770, Ir.-Tur.

Phlomis armeniaca Willd. "boz şavlak", 48, edge of streams, rocky slopes, 1025 m., 10.06.2006, MUÖ 2034, End., LC, Ir.-Tur.

P. capitata Boiss. "çöl çayı", 61, stony slopes, 1280 m., 12.07.2006, MV 9167, End., LC, Ir.-Tur.

P. kurdica Rech. f. "gubel", 62, rocky places, 1150 m., 08.07.2006, SYO 2768, Ir.-Tur.

P. oppositiflora Boiss. \& Hausskn. "has çalba", 32, stony slopes, 1490 m., 12.07.2006, MV 9147, End., LC, Ir.-Tur.

P. pungens Willd. var. hirta Velen, "silvanok", 2, rocky slopes, 1686 m., 08.07.2006, SYO 2786.

Salvia caespitosa Montbret \& Aucher ex Benth. "kırk şalba", 46, rocky places, 1230 m., 05.07.2007, MUÖ 2574, End., LC, Ir.-Tur.

S. candidissima Vahl subsp. candidissima, "galabor", 6, roadsides, rocky slopes, 1320-1690 m., 11.06.2006, MUÖ 2097, Ir.-Tur.

S. hypargeia Fisch. \& C.A. Mey. "siyahot", 42, steppe, 1468 m, 04.06.2007, HA 5638, End., LC, Ir.-Tur.

S. multicaulis Vahl, "kürt reyhanı", 14, roadsides, damp area, 900 m., 14.05.2006, MV 9502, Ir.-Tur.

S. sclarea L. "paskulak", 77, rocky slopes, 900 m., 12.06.2006, MUÖ 2107.

S. staminea Montbret \& Aucher ex Benth. "erkek şalba", 44, rocky places, 1400 m., 15.05.2006, HA 5221, Ir.-Tur.

S. verticillata L. subsp. amasiaca (Freyn \& Bornm.) Bornm. "hart şalbası", 30, steppe, 1031m. 06.07.2006, SYO 2687, Ir.-Tur.

Scutellaria orientalis L. subsp. orientalis, "sarı kaside"41, mixed Juniperus and Quercus area, 1030 m., 16.05.2006, HA 5253, Ir.-Tur.

S. orientalis L. subsp. hausknechtii (Boiss.) J.R. Edm. "saçaklı kaside", 42, mixed Juniperus and Quercus area, 1530 m., 16.05.2006, HA 5271, End., LC, Ir.-Tur.

S. salviifolia Benth. "has kaside", 42, mixed Juniperus and Quercus area, 1030 m., 16.05.2006, HA 5251, End., LC.
Sideritis montana L. subsp. montana, "karaçay", 20, rocky slopes, 1270 m., 04.07.2007, MUÖ 2527, E. Medit.

Stachys annua (L.) L. subsp. annua var. annua, "haciosmanotu", 71, meadows, 1520 m., 02.06.2007, HA 5518.

S. annua (L.) L. subsp. annua var. Iycaonica R. Bhattachaerjee, "hacıosmanotu", 69, stony slopes, 860 m., 03.07.2007, MUÖ 2515, Ir.-Tur.

S. burgsdorffioides (Benth.) Boiss. subsp. ladanoides Hand.-Mazz. "eğin karabaşı", 25, serpentine stony slopes, 1460 m., 13.07.2005, MV 9211, End., NT, Ir.-Tur.

S. cretica L. subsp. anatolica Rech. f. "yağlıkara", 32, stony slopes, 1450 m., 12.07.2006, MV 9126, End., LC, Ir.-Tur.

S. lavandulifolia Vahl, "tüylüçay", 33, rocky places, 1845 m., 05.07.2007, MUÖ 2566.

Teucrium chamaedrys L. subsp. syspirense (K. Koch) Rech.f. "sicakotu", 32, stony slopes, 1490 m., 12.07.2005, MV 9145, Ir.-Tur.

T. leucophyllum Montbret \& Aucher ex Benth. "buldumcuk", 80, above canyon, calcareous rocky places, 1314 m, 02.07.2014, Kandemir n.s., End., LC, Ir.-Tur.

T. multicaule Montbret \& Aucher ex Benth. "haptutan", 17, Quercus fields, 912 m., 14.05.2006, H.A 5024, Ir.-Tur.

T. orientale L. var. puberulens Ekim, "kirveotu", 20, rocky slopes, 1270 m., 04.07.2007, MUÖ 2542, Ir.-Tur.

T. polium L. subsp. polium, "acıyavşan", 32, stony slopes, 1190 m., 12.07.2005, MV 9106.

Thymus cappadocicus Boiss. "taş kekiği", 25, stony slopes, 1460 m., 13.07.2006, MV 9222, End., LC, Ir.-Tur.

T. convolutus Klokov, "eğin kekiği", 35, calcareous rocky places, 1866 m, 11.07.2013, Kandemir 10452, End., EN, Ir.-Tur.

T. hauusknnechtii Velen. "fırat kekiği", 69, stony slopes, 860 m, 03.07.2007, MUÖ 2523, End., NT, Ir.-Tur.

T. leucotrichus Hal. var. leucotrichus, "dağ kekiği", 33, high mountain steppe, 1885 m., 11.06.2006, MUÖ 2058.

T. kotschyanus Boiss. \& Hohen var. kotschyanus, "kekik", 32, stony slopes, 1190 m., 12.07.2006, MV 9109, Ir.-Tur.

T. migricus Klokov \& Des.-Shost. "peynir kekiği", 65, high mountain steppe, 2150 m., 07.07.2007, 
MUÖ 2598, Ir.-Tur.

T. sipyleus Boiss. "sipil kekiği", 61, stony slopes, 1280 m., 12.07.2005, MV 9179.

Ziziphora capitata L. "anuk", 17, Quercus fields, 912 m., 14.05.2006, H.A 5035.

Z. clinopodioides Lam. "dağ reyhanı", 61, stony slopes, 1280 m., 12.07.2005, MV 9170, Ir.-Tur.

Z. persica Bunge, "kara reyhan", 25, serpentine, 1600 m., 03.06.2007, HA 5530, Ir.-Tur.

\section{LILIACEAE / ZAMBAKGILLER}

Fritillaria imperialis L. "ağlayan gelin", 44, rocky places, 1400 m., 15.05.2006, HA 5250, Ir.-Tur.

F. pinardii Boiss. "mahcup lale", 6, rocky places, 1680 m., 13.04.2006, MV 9341, Ir.-Tur.

Gagea taurica Steven, "bozkır yıldızı", 34, rocky places, 1650 m., 14.04.2006, MV 9375, Ir.-Tur.

Tulipa armena Boiss. var. armena, "dağ lalesi", 5, rocky places, 915 m., 13.04.2006, MV 9332, Ir.-Tur.

\section{LINACEAE / KETENGILLER}

Linum bienne Mill. "deli keten", 33, rocky places, 1900 m., 02.06.2007, HA 5496.

L. catharticum L. "arsız keten", 62, steppe, 1200 m., 08.07.2006, SYO 2766.

L. flavum L. subsp. scabrinerve (P.H. Davis) P.H. Davis, "kaba çimit", 42, steppe, 1468 m., 04.06.2007, HA 5632, End., LC, Ir.-Tur.

L. mucronatum Bertol. subsp. mucronatum, "sarı keten", 34, rocky slopes, 1400-1640 m., 11.06.2006, MUÖ 2071.

L. nodiflorum L. "yaban keten", 47, rocky slopes, 858 m., 27.06.2006, MUÖ 2105, E. Medit.

\section{LYTHRACEAE / AKLAROTUGILLER}

Lythrum salicaria $L$, "hevhulma", 4, serpentine stony slopes, 915 m., 13.07.2005, MV 9185, EuroSib.

\section{MALVACEAE / EBEGÜMECIGILLER}

Alcea apterocarpa (Fenzl) Boiss. "gülfatma", 48, rocky slopes, 1025 m., 10.06.2006, MUÖ 2031, Ir.Tur.

A. digitata (Boiss.) Alef. "boylu hatmi", 6 roadsides, rocky slopes, 1090 m., 12.06.2006, MUÖ 2102, Ir.-Tur.

A. excubita Iljin, "amervans", 69, rocky slopes, 860 m., 03.07.2007, MUÖ 2522, Ir.-Tur.

Malva neglecta Wallr. "çobançöreği", 33, high mountain steppe, 1885 m., 11.06.2006, MUÖ 2067.

* Tilia rubra DC. subsp. caucasica (Rupr.) V. Engl. "felamur", 13, cultivated area, 15.04.2006, MV 9510, Euro-Sib.

MORACEAE / DUTGILLER
Morus alba L. "ak dut", 4, serpentine stony slopes, 1060 m., 13.07.2005, MV 9235.

\section{OLEACEAE / ZEYTINGILLER}

Jasminum fruticans L. "boruk", 4, serpentine stony slopes, 915 m., 13.07.2005, MV 9199, E. Medit.

Fraxinus angustifolia Vahl. subsp. angustifolia, "sivri dişbudak", 14, roadsides, 1270 m., 14.05.2006, MV 9567.

F. angustifolia Vahl. subsp. syriaca (Boiss.) Yalt., "suriye dişbudağı", 4, roadsides, 1138 m., 08.07.2006, SYO 2775, Ir.-Tur.

Syringa vulgaris L. "leylak", 54, roadsides, 861 m., 04.06.2007, HA 5623.

\section{ONAGRACEAE / YAKIOTUGILLER}

Epilobium hirsutum L. "hasanhüseyin çiçeği", 63, damp area, 1360 m., 14.09.2007, SYO 2973.

\section{ORCHIDACEAE / SALEPGILLER}

Cephalanthera damasonium (Mill.) Druce, "orman kuşçuğu", 55, Quercus fields, 1287-1313 m., 14.05.2006, HA 5080, Euro-Sib.

C. kotschyana Renz \& Taubenheim, "koç salebi", 17, Quercus fields, 912 m., 14.05.2006, HA 5031, End., LC.

C. Iongifolia (L.) Fritsch, "kuğu salebi", 42, mixed Juniperus and Quercus area, 1530 m., 16.05.2006, HA 5266, Euro-Sib.

Dactylorhiza iberica (M. Bieb. ex Willd.) Soó, "kırım salebi", 61, edge of stream, 1500 m, 07.07.2007, MUÖ 2614, E. Medit.

D. osmanica (Klinge) P.F. Hunt \& Summerh. var. osmanica, "osmanlı salebi", 14, damp area, 1270 m., 14.05.2006, MV 9455, End., LC, Ir.-Tur.

Ophrys oestrifera M. Bieb. subsp. oestrifolia, "sinek salebi", 42, mixed Juniperus and Quercus area, 1530 m., 16.05.2006, HA 5078.

O. phrygia H. Fleischm. \& Bornm. "yunus salebi", 4, meadows, 1040 m., 16.05.2006, HA 5305, Ir.Tur.

Orchis mascula (L.) L. subsp. pinetorum (Boiss. \& Kotschy) G. Camus, "çam salebi", 42, mixed Juniperus and Quercus area, 1530 m., 14.05.2006, HA 5274, E. Medit.

0. punctulata Steven ex Lindley, "selef", 42, mixed Juniperus and Quercus area, 1530 m., 16.05.2006, HA 5267, E. Medit.

\section{OROBANCHACEAE / CANAVAROTUGILLER}

Bungea trifida (Vahl) C.A. Meyer, "üç kernekotu", 56, meadows, 1715 m., 09.07.2006, SYO 2796, Ir.Tur. 
Orobanche caryophyllacea Sm. "kokulu süpürgeotu", 42, Juniperus and Quercus mixed area, 1530 m., 16.05.2006, HA 5268.

*Parentucellia latifolia (L.) Caruel subsp. flaviflora (Boiss.) Hand.-Mazz, "sarı üçdilotu", 49, Quercus fields, 1200 m., 15.05.2006, HA 5194.

\section{PAEONIACEAE / AYIGÜLÜGILLER}

Paeonia arietina G. Anderson, "şakayık", 35, stony places, 1875 m, 07.07.2006, SYO 2735.

\section{PAPAVERACEAE / HAŞHAŞGILLER}

Corydalis alpestris C.A. Mey. "gök kazgagası", 6, roadsides, 1400 m., 13.04.2006, MV 9365, EuroSib.

Fumaria asepala Boiss. "ak şahtere", 17, Quercus fields, 912 m., 14.05.2006, HA 5025, Ir.-Tur.

F. officinalis L. subsp. officinalis, "şahtere", 55, Quercus fields, 1287-1313 m., 14.05.2006, HA 5071. Hypecoum pseodograndiflorum Petrovic, "hıdrellezotu", 17, Quercus fields, 912 m., 14.05.2006, HA 5030.

Glaucium leiocarpum Boiss. "gavurhaşhaşı", 49, Quercus fields, 1200 m., 15.05.2006, HA 5199.

G. acutidentatum Hausskn. \& Bornm. "tavukgötü", 1, stony slopes, 1600 m., 13.07.2006, MV 9249, End., LC, Ir.-Tur.

Papaver acrochaetum Bornm. "pıtçık", 28, rocky slopes, 1400 m., 02.07.2007, MUÖ 2496, Ir.-Tur.

*P. argemone L. subsp. argemone, "kum haşhaşı", 55, Quercus fields, 1287-1313 m., 14.05.2006, HA 5110.

P. cylindricum Cullen, "kabırcık", 62, rocky places, 960 m., 08.07.2006, SYO 2773, Ir.-Tur.

P. dubium L. subsp. dubium, "köpekyağı", 17, Quercus fields, 912 m., 14.05.2006, HA 5056.

P. fugax Poir. var. fugax, "gelingülü", 25, serpentine, 1600 m., 03.06.2007, HA 5546.

P. glaucum Boiss. \& Hausskn. ex Boiss. "şekşekik", 14, roadsides, 1270 m., 14.05.2006, MV 9505, Ir.Tur.

P. persicum Lindl. subsp. persicum Boiss., "acem gelinçiçeği", 30, steppe, 940 m., 13.09.2007, SYO 2965.

P. rhoeas L. 55, rocky slopes, "gelincik", 1300 m., 14.05.2006, HA 5115.

P. triniifolium Boiss. "titrekızım", 20, stony slopes, 1270 m., 04.07.2007, MUÖ 2538, End., LC, Ir.-Tur. PHYLLANTHACEAE / DUVARNOHUTUGILLER Andrachne telephioides L. "duvarnohutu", 49, Quercus fields, 1200 m., 15.05.2006, HA 5186.

PLANTAGINACEAE / SINIROTUGILLER
Anarrhinum orientale Benth. "süpürgeotu", 28, rocky slopes, 1400 m., 02.07.2007, MUÖ 2461, Ir.Tur.

Globularia trichosantha Fisch. \& C.A. Mey. subsp. trichosantha, "köse yayılımı", 6, rocky slopes, 1400 m., 13.04.2006, MV 9362.

Hippuris vulgaris L. "kurakkuyruğu", 76, slough, 1724 m., 15.09.2007, SYO 2989.

*Linaria armeniaca Chav. "doğu nevruzotu", 55, Quercus fields, 1287-1313 m., 14.05.2006, HA 5093, Ir.-Tur.

L. genistifolia (L.) Mill. subsp. genistifolia, "som nevruzotu", 44, rocky places, 1400 m., 15.05.2006, HA 5227, Euro-Sib.

Veronica anagallis-aquatica L. "sudegemesi", 13, meadows, 900 m., 15.04.2006, MV 9558a.

V. bozakmanii M.A. Fisch. "bozakman mavişi", 13, meadows, 900 m., 15.04.2006, MV 9558, Ir.-Tur.

V. macrostachya Vahl subsp. mardinensis (Bornm.) M.A. Fisch. "mardin mavişi", 55, Quercus fields, 1287-1313 m., 14.05.2006, HA 5081, End., VU, Ir.-Tur.

V. multifida L. "devesabunu", 8, under Juniperus oxycedrus, 964 m., 15.05.2006, HA 5181, Ir.-Tur. PLATANACEAE / ÇINARGILLER

Platanus orientalis L. "çınar", 14, roadsides, 1270 m., 14.05.2006, MV 9493.

PLUMBAGINACEAE / KARDIKENIGILLER

Acantholimon acerosum (Willd.) Boiss. subsp. acerosum, "pisik keveni", 69, stony slopes, 860 m., 03.07.2007, MUÖ 2520, Ir.-Tur.

Limoniopsis owerinii (Boiss.) Lincz. "eğin kuduzotu", 51, kalker kayalık, 1570 m., 05.07.2005, Kandemir 7028, Ir.-Tur.

\section{POACEAE / BUĞDAYGILLER}

Alopecurus vaginatus (Willd.) Kunth, "benekli tilkikuyruğu", 33, calcareous rocky places, 1850m., 17.05.2006, HA 5326.

Bromus japonicus Thunb. subsp. japonicus, "iyeotu", 60, edge of stream, 1500 m, 07.07.2007, MUÖ 2613.

Dactylis glomerata L. subsp. hispanica (Roth) Nyman, "kıllı domuz ayrığı", 14, roadsides, 1270 m., 14.05.2006, MV 9524.

Heteranthelium piliferum (Banks. \& Sol.) Hochst. "çorakarpası", 38, roadsides, 1400-1640 m., 11.06.2006, MUÖ 2072, Ir.-Tur.

Hordeum bulbosum L. "boncuk arpa", 14, roadsides, 1270 m., 14.05.2006, MV 9564.

Koelaria pyramidata (Lam.) P. Beauv. "kırnal", 
65, high mountain steppe, $2150 \mathrm{~m}$, 07.07.2007, MUÖ 2602, Euro-Sib.

Melica ciliata L. subsp. ciliata, "kirpikli inci", 32, stony slopes, 1490 m., 12.07.2005, MV 9134.

M. persica Kunth subsp. jacquemontii (Decne. ex Jacqem.) P.H. Davis, "çayır inciotu", 33, steppe, 1875 m., 07.07.2006, SYO 2725, Ir.-Tur.

Oryzopsis holciforme (M. Bieb.) Hack. var. holciformis, "kadife pirinçotu", 14, steppe, 1270 m., 14.05.2006, MV 9509.

Pennisetum orientale Rich. "fıskiyeotu", 25, serpentine slopes, 1460 m., 13.07.2005, MV 9208 , Ir.-Tur.

Poa bulbosa L. "yumrulu salkım", 28, rocky slopes, 1400 m.,02.07.2007, MUÖ 2472.

P. pratensis $L$. "çayır salkımotu", 34, rocky places, 1630 m., 02.06.2007, HA 5440a.

P. sterilis M. Bieb. "köse salkımotu", 28, rocky slopes, 1400 m.,02.07.2007, MUÖ 2471.

P. trivialis L. "kaba salkımotu", 32, stony slopes, 1490 m.,12.07.2005, MV 9134a.

Stipa ehrenbergiana Trin. \& Rupr. "sorguçotu", 8, under Juniperus oxycedrus, 964 m., 15.05.2006, HA 5184, Ir.-Tur.

Taeniatherum caput-medusae (L.) Nevski subsp. crinitum (Schreb.) Melderis, "kılçık arpası", 69, stony slopes, 860 m, 03.07.2007, MUÖ 2514, Ir.Tur.

\section{POLYGALACEAE / SÜTOTUGILLER}

Polygala pruinosa Boiss. subsp. pruinosa, "puslu sütotu", 67, meadows and rocy places, $950 \mathrm{~m}$. 03.06.2007, HA.5577.

\section{POLYGONACEAE / MADIMAKGILLER}

Rumex acetosella L. "kuzukulağı", 34, rocky slopes, 1400-1640 m., 11.06.2006, MUÖ 2073.

\section{PRIMULACEAE / ÇUHAÇiçEĞiGiLLER}

Androsace maxima L. "tavukkursağı", 67, meadows and rocky places, 950 m., 03.06.2007, HA 5590.

\section{RANUNCULACEAE / DÜĞÜNÇiçEĞiGíLLER}

Adonis flammea Jacq. "cinlalesi", 17, Quercus fields, 912 m., 14.05.2006, HA 5026.

Aquilegia olypica Boiss. "hasekiküpesi", 61, rocky places, 1310 m., 17.05.2006, HA 5335.

Consolida scleroclada (Boiss.) Schrödinger var. rigida (Freyn \& Sint.) P.H. Davis, "sert mahmuz", 46, rocky places, 1230 m., 05.07.2007, MUÖ 2570. Delphinium albiflorum DC. "ak hezaren", 37, steppe, 1671 m., 07.07.2006, SYO 2740.

Eranthis hyemalis (L.) Salisb. "sarıkokulu", 33, meadows, 1910 m., 05.05.2005, Kandemir 6882. Nigella oxypetala Boiss. "fırat çörekotu", 28, rocky slopes, 1400 m., 02.07.2007, MUÖ 2495, Ir.Tur.

N. nigellastrum (L.) Willk. "cüccem", 28, rocky slopes, 1170 m., 02.07.2007, MUÖ 2465.

Ranunculus. constantinopolitanus (DC.) d'Urv. "kağıthane çiçeği", 34, rocky slopes, 1650 m., 14.04.2006, MV 9381.

R. arygreus Boiss. "çitemik", 55, steppe, 1300 m., 14.05.2006, HA 5113.

R. arvensis L. "mustafaçiçeği", 17, Quercus fields, 912 m., 14.05.2006, HA 5020

R. pinardii (Steven) Boiss. "gazyağıotu", 34, rocky places, 1670 m., 17.05.2006, HA 5320, Ir.-Tur.

R. kochii Ledeb. "karçiçeği", 34, rocky slopes, 1650 m., 14.04.2006, MV 9378, Ir.-Tur.

Thalictrum isopyroides C.A. Mey. "karakatranotu", 50, rocky places, 1200 m., 16.05.2006, HA 5306, Ir.-Tur.

\section{RESEDACEAE / GERDANLIKGILLER}

Reseda armena Boiss. var. scabridula Abdullah \& de Wit, "has gerdanlık", 23, serpentine, 1467 m., 05.07.2005, Kandemir 7008, End., EN. Ir.-Tur.

R. Iutea L. var. Iutea, "muhabbet çiçeği", 8 , under Juniperus oxycedrus, 964 m., 15.05.2006, HA 5178.

\section{RHAMNACEAE / CEHRIGILLER}

Paliurus spina-christii P. Mill. "karaçalı", 24, steppe, 990 m., 15.05.2006, HA 5671.

Rhamnus cathartica L. "akdiken", 33, rocky places, 1182 m., 23.09.06, SYO 2830, Euro-Sib.

\section{ROSACEAE / GÜLGILLER}

Cerasus mahaleb (L.) Mill. var. mahaleb, "mahlep", 13, meadows, 900 m., 15.04.2006, MV 9395.

C. microcarpa (C.A. Meyer) Boiss. subsp. tortuosa (Boiss. \& Hausskn.) Browicz, "sarıdağ kirazı", 45, stony slopes, 1150 m., 12.07.2005, MV 9159, Ir.-Tur. Cotoneaster nummularius Fisch. \& C.A. Mey. "dağ muşmulası", 7, steppe, 1485 m., 24.09.06, SYO 2838.

Crataegus orientalis Pallas ex M. Bieb. var. orientalis, "alıç", 6, rocky places, 24.09.2006, SYO 2834.

C. orientalis Pallas ex M. Bieb. var. szowitsii (Pojark.) K.I.Chr. "koyun alıcı", 32, rocky places, 1182 m., 23.09.2006, SYO 2826, Ir.-Tur.

C. meyeri Pojark, "roğuk", 63, rocky places, 1364 m., 25.09.2006, SYO 2842.

*Fragaria vesca L. "dağ çileği", 13, meadows, 900 
m., 15.04.2006, MV 9396, Euro-Sib.

Geum urbanum $\mathrm{L}$. "meryemotu", 44, rocky places, 1400 m., 15.05.2006, HA 5232, Euro-Sib.

Malus sylvestris (L.) Mill. subsp. orientalis (Uglitzk.) Browicz var. orientalis, "ekşi elma", 13, meadows, 900 m., 15.04.2006, MV 9496.

*Potentilla aff. micrantha Ramond ex DC. "cüce parmakotu", 65, high mountain steppe, $2150 \mathrm{~m}$. 07.07.2007, MUÖ 2599.

P. recta L. "su parmakotu", 48, edge of stream, rocky slopes, 1025 m., 10.06.2006, MUÖ 2035.

P. reptans L. "reşatınotu", 43, cultivated area, 1277 m., 10.06.2006, MUÖ 2045.

P. speciosa Willd. "kaya parmakotu", 63, rocky slopes, 1360 m., 14.09.2007, SYO 2978.

P. supina L. "yer parmakotu", 69, stony slopes, 860 m, 03.07.2007, MUÖ 2507.

Prunus divericata Ledeb. var. divericata, "yunus eriği", 44, roadsides, 1252 m., 06.07.2006, SYO 2702.

Pyrus elaeagnifolia Pall. subsp. elaeagnifolia, "ahlat", 44, roadsides, 1252 m., 06.07.2006, SYO 2701.

P. elaeagnifolia Pall. subsp. kotschyana (Boiss.) Browicz, "dağ armudu", 64, roadsides, 1912 m., 25.09.2006, SYO 2849.

Rosa foetida J. Herrm. "acemsarısı", 13, meadows, 900 m., 15.04.2006, MV 9555, Ir.-Tur.

R. orientalis A. Dupont ex DC. "askergülü", 38, roadsides, 1875 m., 07.07.2006, SYO 2713, Ir.-Tur.

Rubus sanctus Schreb. "böğürtlen", 30, roadsides, 1031m., 06.07.2006, SYO 2682.

Sangiosorba minor Scop. subsp. minor, "çayırdüğmesi", 13, meadows, 900 m., 15.04.2006, MV 9533.

Spiraea crenata L. subsp. crenata, "keçisakalı", 20, rocky slopes, 1270 m., 04.07.2007, MUÖ 2541.

Sorbus torminalis (L.) Crantz var. torminalis, "pitlicen", 30, roadsides, 1031m., 06.07.2006, SYO 2688.

S. umbellata Fritsch, "geyikelması", 1, stony slopes, 1600 m., 13.07.2005, MV 9253.

RUBIACEAE / KÖKBOYAGILLER

Asperula arvensis L. "tarla belumotu", 17, Quercus fields, 912 m., 14.05.2006, HA 5058, E. Medit.

A. involucrata Wahlenb. "akça belumotu", 16, meadows, 1084 m., 15.05.2006, HA 5130, EuroSib.

Callipeltis cucullaris (L.) Steven, "nermik", 34, rocky places, 1630 m, 02.06.2007, HA 5472, Ir.Tur.

Crucianella gilanica Trin. subsp. kotschyi (Ehrend.) Ehrend. "eğin haçotu", 28, rocky slopes, 1400 m, 02.07.2007, MUÖ 2490, Ir.-Tur.

Cruciata taurica (Pall. ex Willd.) Ehrend. "kırım güzeli", 11, rocky slopes, 1150 m., 15.05.2006, HA 5211, Ir.-Tur.

Galium ceratocarpon Boiss. "kırkgöz iplikçiği", 11, rocky slopes, 1150 m., 15.05.2006, HA 5209, End., NT, Ir.-Tur.

G. hypoxylon Ehrend. \& Schönb.- Tem. "keşiş iplikçiği", 33, steppe, 1885 m., 11.06.2006, MUÖ 2059, End., VU, Ir.-Tur.

G. incanum Sm. subsp. elatius (Boiss.) Ehrend. "gür iplikçik", 29, rocky slopes, 1500 m., 02.07.2007, MUÖ 2504, Ir.-Tur.

G. margaceum Ehrend. \& Schönb.-Tem. "saman iplikçiği", 34, rocky places, 1630 m., 02.06.2007, HA 5468, End., LC.

G. spurium L. subsp. spurium, "arsız iplikçik", 39, rocky places, 870 m., 15.05.2006, HA 5149, EuroSib.

\section{SALICACEAE / SÖĞÜTGILLER}

Populus nigra L. subsp. nigra, "karakavak", 14, roadsides, 1270 m., 14.05.2006, MV 9498.

P. tremula L. subsp. tremula, "titrek kavak", 15, roadsides, 23.09.2006, SYO 2817, Euro-Sib.

Salix x fragilis L. "gevrek söğüt", 13, cultivated area, 900 m., 15.04.2006, MV 9529, Euro-Sib.

S. pseudomedemii E. Wolf, "kara söğüt", 14, roadsides, 1270 m., 14.05.2006, MV 9548, EuroSib.

\section{SANTALACEAE / GÜVELEKGILLER}

Chrysothecium cilicicum (Hausskn. ex Bornm.) Hendrych, "Toros güveleği"33, rocky places, 1900 m., 02.06.2007, HA 5504, End., NT, E. Medit.

\section{SAPINDACEAE / AKÇAAĞAÇGILLER}

*Acer monspessulanum L. subsp. microphyllum (Boiss.) Bornm. "buruk akçaağaç", 11, rocky places, 1150 m, 15.05.2006, HA 5205.

A. tataricum L. subsp. tataricum, "tatar akçaağacı", 12, cultivated area, 900 m, MV 9528.

SCROPHULARIACEAE / SIRACAOTUGILLER

Verbascum calycosum Hausskn ex Murb. "kalan sığırkuyruğu", 23, serpentine, 1502 m., 12.06.2004, Kandemir 6086, End., CR, Ir.-Tur.

V. cherianthifolium Boiss. var. asperulum (Boiss.) Murb. "bozkulak", 25, serpentine, 1000 m., 15.05.2006, HA 5782. 
V. leiocarpum Murb. "gürbüz sığırkuyruğu", 52, rocky places, 890 m., 06.07.2007, MUÖ 2561, End., VU, Ir.-Tur.

V. orientale (L.) All. Subsp. orientale, "ibrahimotu", 28, rocky slopes, 1400 m., 02.07.2007, MUÖ 2452, E. Medit.

V. tuna-ekimii Karavel. "ekim sığırkuyruğu", 6, rocky slopes, 1400 m., 11.06.2006, MUÖ 2096, End., VU, Ir.-Tur.

Scrophularia libanotica Boiss. subsp. armena R.R. Mill. "kayaçekemi", 49, Quercus fields, 1200 m., 15.05.2006, HA 5387, End., NT, Ir.-Tur.

Scrophularia subaeqiloba Lall, "munzur sıracası", 25, 950 m, serpentine. 19.05.2013, Kandemir 10320, End, VU, Ir.-Tur.

S. umbrosa Dumort, "su kestereotu", 62 roadsides, 08.07.2006, SYO 2765, Euro-Sib.

S. xanthoglossa Boiss. var. decipiens (Boiss. \& Kotschy) Boiss. "serkele", 56, rocky places, 1715 m., 09.07.2006, SYO 2798, Ir.-Tur.

\section{SOLANACEAE / PATLICANGILLER}

Hyoscyamus niger L. "banotu", 5, rocky places, 1343 m., 06.06.2007, HA 5668.

Solanum dulcamara L. "sofur", 12, roadsides, 970 m., 08.07.2006, SYO 2764, Euro-Sib.

\section{TAMARICACEAE / ILGINGILLER}

Tamarix smyrnensis Bunge, "Ilgın", 25, serpentine, 1600 m., 03.06.2007, HA 5551.

T. tetrandra Pall. ex M. Bieb. emend. Willd. "gezik", 69, rocky slopes, 860 m., 03.07.2007, MUÖ 2517.

THYMELAEACEAE / SIYIRCIKGILLER

Daphne oleoides Schreb. subsp. oleoides, "gövçek", 34, rocky places, 1630 m., 02.06.2007, HA 5480.

\section{TYPHACEAE / SAZGILLER}

Typha shuttleworthii W.D.J. Koch \& Sond. "puf sazı", 76, wetlands, 1724 m., 15.09.2007, SYO 2988.

\section{ULMACEAE / KARAAĞAÇGILLER}

Ulmus minor Mill. "ova karaağacı", 63, roadsides, 1364 m., 25.09.2006, SYO 2844.

\section{URTICACEAE / ISIRGANGILLER}

Parietaria judaica L. "duvarfesleğeni", 48, rocky slopes, 1025 m., 10.06.2005, MUÖ.2025.

\section{VIOLACEAE / MENEKŞEGILLER}

Viola occulta Lehm. "saklı menekşe", 49, Quercus fields, 1200 m., 15.05.2006, HA 5188.

V. odorata L. "kokulu menekşe", 13, meadows, 900 m., 15.04.2006, MV 9390.

XANTHORRHOEACEAE / ÇIRIŞGILLER
Asphodeline damascena (Boiss.) Baker subsp. damascena, "çekiçlik", 44, rocky places, 1400 m., 15.05.2006, HA 5242, Ir.-Tur.

A. tenuior (Fisch. ex M. Bieb.) Ledeb subsp. tenuiflora (K. Koch) Tuzlacı var. puberulanta E. Tuzlacı, "tesbihçik", 23, serpentine, 1467 m., 05.07.2005, Kandemir 7019, End., VU, Ir.-Tur.

Eremurus spectabilis M. Bieb. "çiriş", 44, rocky places, 1400 m., 15.05.2006, HA 5244, Ir.-Tur.

\section{Acknowledgments}

Authors would like to thank to the Scientific and Technical Research Council of Turkey for its financial support (CAYDAG-105Y016); to Prof. Dr. Tuna Ekim and Prof. Dr. Mecit Vural for their helps during identification of the some specimens; to project coordinator Prof. Dr. Ali Demirsoy; to the Kemaliye people; and especially to Şevket Gültekin because of his helps to our field studies.

\section{References}

1. Anonim, Kemaliye İlçesi Iklim Verileri (1984-1990), Meteoroloji Genel Müdürlüğü, Araştırma ve Bilgi İşlem Daire Başkanlığı, Ankara, 2005a.

2. Anonim, Genel Nüfus Sayımı, Nüfusun Sosyal ve Ekonomik Nitelikleri, 24 Erzincan, T. C. Başbakanlık Devlet İstatistik Enstitüsü, Ankara, 2005b.

3. N. Özgül, Munzur Dağlarının Jeolojisi. Maden ve Tetkik Arama Genel Müdürlüğü. Derleme Raporu No:6995, Ankara, 1981.

4. Anonim, Erzincan İli Arazi Varlığı, T. C. Başbakanlık Köy Hizmetleri Genel Müdürlüğü Yayınları, Rapor No: 24, Erzincan, 2000

5. Y. Akman, İklim ve Biyoiklim, Palme Yayın. Ankara, 1990.

6. Anonim, Erzincan Iklim Verileri, Devlet Meteoroloji İşleri Genel Müdürlüğü, Ankara, 2007.

7. P.H. Davis (ed), Flora of Turkey and the East Aegean Islands, Vol. 1-9, Edinburgh: Edinburgh University Press, 1965-1985.

8. P.H. Davis, K. Tan, R.R. Mill, R.R. (eds), Flora of Turkey and the East Aegean Islands, v: 10, Edinburgh: Edinburgh University Press, 1988.

9. A. Güner, N. Özhatay, T. Ekim, K.H.C. Başer (eds), Flora of Turkey and the East Aegean Islands, vol: 11, Edinburgh: Edinburgh University Press, 2000.

10. K.H. Rechinger (ed), Flora Iranica, Graz Austria: Akademische Druck-u. Verlagsanstalt, 1965-1977.

11. E. Guest, Flora of Iraq, v: 4, Iraq: Published by Ministry of Agriculture and Agrarian Reform, 1980.

12. M. Zohary (ed), Flora Palaestina, vol: 1-4 (Text and Plate), The Israel Academy of Science and Humanities, Jerusalem Academic Press, (1966-1986).

13. G.T. Tutin, V.H. Heywood, N.A. Burges, D.H. Valentine, S.M. Walters \& D.A. Webb (eds), Flora Europaea. vol: 1-5. Cambridge: Cambridge University Press, 19641980.

14. B.K. Shishkin, V.L. Komarov, Flora of USSR, Leningrad, 1933-1964.

15. A. Güner, S. Aslan, T. Ekim, M. Vural, M.T. Babaç (eds), Türkiye Bitkileri Listesi (Damarlı Bitkiler). İstanbul, Turkey: Nezahat Gökyiğit Botanik Bahçesi ve Flora Araştırmaları Derneği Yayını. İstanbul. 2012. 
16. T. Ekim, M. Koyuncu, M. Vural, H. Duman, Z. Aytaç (eds), Türkiye Bitkileri Kırmızı Kitabı (Pteridophyta ve Spermatophyta), Ankara: Türkiye Tabiatını Koruma Derneği ve Van 100. Yıl Üniversitesi Yayını, 2000.

17. IUCN, IUCN Red List Categories: Version 3.1. Prepared by the IUCN Species Survival Commission, IUCN Gland, Switzerland and Cambridge, UK, 2001.

18. R.K. Brummitt, C.E. Powell (eds), Authors of Plant Names, Kew, The Royal Botanic Gardens, 1992.

19. Ş. Yıldırımlı, Flora of Munzur Dağları (ErzincanTunceli), Ot Sis. Bot. Derg. 2 (1995) 1-78.

20. H.A. Akpulat, N. Çelik, Sivas-Sıcak Çermik Arası Florası, C.Ü. Fen Bilimleri Dergisi, 23 (2002) 1-15.

21. i. Türkoğlu, Ş. Civelek, Karga Dağının (Elazığ) Florası, F.Ü. Fen ve Mühendislik Bilimleri Dergisi, 17 (2005) 370-399.

22. E. Dönmez, Gövdeli Dağı (Kayseri-Sivas) Florası, C.Ü. Fen Bilimleri Enstitüsü, Sivas, 1999.

23. J. Donner, Distribution Maps to P.H. Davis, Flora of Turkey 1-10, Linzer biol. Breitr, Linz, 1990.

24. S. Yıldırımlı, The chorology of the Turkish species of Apiaceae family, Ot Sis. Bot. Derg., 4 (1997) 105-128.

25. S.. Yıldırımlı, The chorology of the Turkish species of Asteraceae family, Ot. Sis. Bot. Derg., 6 (1999) 75-123.

26. S.. Yıldırımlı, The chorology of the Turkish species of Boraginaceae family, Ot. Sis. Bot. Derg., 7 (2000) 257-272.

27. Ş. Yıldırımlı, The chorology of the Turkish species of Brassicaceae, Buddlejaceae and Buxaceae families, Ot. Sis. Bot. Derg., 8 (2001a) 141-171.

28. Ş. Yıldırımlı, The chorology of the Turkish species of Cactaceae, Callitrichaceae, Calycanthaceae and Campanulaceae families, Ot. Sis. Bot. Derg., 8 (2001b) 157-171.

29. Ş. Yıldırımlı, The chorology of the Turkish species of Caryophyllaceae, Casuarinaceae, Celestraceae, Ceratophyllaceae and Cercidiphyllaceae families, Ot. Sis. Bot. Derg., 9 (2002) 175-199.

30. Ş. Yıldırımlı, The chorology of the Turkish species of Chenopodiaceae, Cistaceae, Convolvulaceae, Cornaceae and Corylaceae families, Ot. Sis. Bot. Derg. 10 (2003a) 203-215.

31. Ş. Yıldırımlı, The chorology of the Turkish species of Crassulaceae, Cucurbitaceae, Cuscutaceae and Cynocrambaceae families, Ot. Sis. Bot. Derg., 10 (2003b) 249-263.

32. S.. Yıldırımlı, The chorology of the Turkish species of Fabaceae, Ot. Sis. Bot. Derg., 12 (2005) 117-170.

33. Ş. Yıldırımlı, The chorology of the Turkish species of Lamiaceae family, Ot. Sis. Bot. Derg., 14 (2008a) 151198.

34. S. Yıldırımlı, The chorology of the Turkish species of Magnoliaceae, Malvaceae, Meliaceae, Menyanthaceae, Molluginaceae, Moraceae, Morinaceae, Myrtaceaei Nyctaginaceae, Nymphaeaceae and Nyssaceae families, Ot. Sis. Bot. Derg., 15 (2008b) 150-164.
35. Ş. Yıldırımlı, The chorology of the Turkish species of Paeoniaceae, Papaveraceae, Parnassiaceae, Passifloraceae, Pedaliaceae, Phytolaccaceae, Piperaceae, Pittosporaceae, Plantaginaceae and Platanaceae families, Ot. Sis. Bot. Derg., 16 (2009) 171-186.

36. Ş. Yıldırımlı, The chorology of the Turkish species of Rafflesiaceae and Ranunculaceae families, Ot. Sis. Bot. Derg., 17 (2010) 199-223.

37. Ş. Yıldırımlı, The chorology of the Turkish species of Rosaceae family, Ot Sis. Bot. Derg. 18 (2011a) 191-235.

38. Ş. Yıldırımlı, The chorology of the Turkish species of Rubiaceae and Rutaceae families, Ot. Sis. Bot. Derg., 18 (2011b) 173-204.

39. Ş. Yıldırımlı, The chorology of the Turkish species of Scrophulariaceae family, Ot. Sis. Bot. Derg., 19 (2012) 151-211.

40. Ş. Yıldırımlı, The chorology of the Turkish species of Tamaricaceae, Theaceae, Thymelaeaceae, Tiliaceae, Trapaceae, Tropaeolaceae, Ulmaceae and Urticaceae families, Ot. Sis. Bot. Derg., 20 (2013) 157-170.

41. Ş. Yıldırımlı, The chorology of the Turkish flowering Monocotyledones species of Alismataceae, Amaryllidaceae, Araceae, Arecaceae, *Bromeliaceae, Butomaceae, *Cannaceae, *Commelinaceae and Cymodoceaceae families, Ot. Sis. Bot. Derg., 21 (2014a) 143-162.

42. S. Yıldırımlı, The chorology of the Turkish flowering Monocotyledones species of Dioscoreaceae, Hydrocharitaceae, Iridaceae, Juncaginaceae and Lemnaceae families, Ot. Sis. Bot. Derg., 21 (2014b) 119145.

43. S. Yıldırımlı, The chorology of the Turkish tepaloid Monocotyledones species of Liliaceae Juss. s.I. family, Ot. Sis. Bot. Derg., 22 (2015) 67-119.

44. S. Yıldırımlı, The chorology of the Turkish non tepaloid Monocotyledones species of Cyperaceae Juss., Juncaceae Juss. and Poaceae Barnhart (Gramineae) families, Ot. Sis. Bot. Derg., 24 (2017) 173-235.

45. A. Kandemir, S. Makbul, Erzincan Yöresinde Yayılış Gösteren Bazı Nadir Bitki Türleri Üzerine Gözlemler, Erzincan Eğitim Fakültesi Dergisi, 6 (2004) 37-49.

46. A. Kandemir, The Rediscovery of Some Taxa Thought to Have Been Extinct in Turkey, Turk. J. of Bot., 33 (2009) 113-122.

47. A. Kandemir, M. Korkmaz, S. Karacan, Bilim dünyası için yeni sinonimler ve yeni kombinasyon, Bağbahçe Bilim Derg., 1 (2014) 82-94.

48. A. Kandemir, C. Sevindi, M. Korkmaz, Ş. Çelikoğlu, Erzincan (Türkiye)'a özgü endemic bitki taksonlarının IUCN tehdit kategorileri, Bağbahçe Bilim Derg., 2 (2015) 43-65.

49. Ş. Yıldırımlı, Ö. Kılıç, New infrageneric taxa and species of Aethionema W.T. Aiton (Brassicaceae) and their current key from Turkey, Ot. Sis. Bot. Derg., 23 (2016) 1-66. 
